\title{
THE BRAUER GROUP AND THE SECOND ABEL-JACOBI MAP FOR 0-CYCLES ON ALGEBRAIC VARIETIES
}

\author{
GERALD E. WELTERS
}

\begin{abstract}
This paper focuses on the connection between the Brauer group and the 0-cycles of an algebraic variety. We give an alternative construction of the second l-adic AbelJacobi map for such cycles, linked to the algebraic geometry of Severi-Brauer varieties on X. This allows us then to relate this Abel-Jacobi map to the standard pairing between 0-cycles and Brauer groups (see [M], [L]), completing results from [M] in this direction. Second, for surfaces, it allows us to present this map according to the more geometrical approach devised by M. Green in the framework of (arithmetic) mixed Hodge structures (see [G]).

Needless to say, this paper owes much to the work of U. Jannsen and, especially, to his recently published older letter [J4] to B. Gross.
\end{abstract}

\section{Contents}

1. Introduction

2. The map $\Delta_{n}^{1}$

3. Construction of $\Delta_{n}^{2}$.

4. A cohomological description of $\alpha_{Z}$ 463

5. Comparison with the $l$-adic Abel-Jacobi map $d_{n}^{2}$ 471

6. A comparison with M. Green's method 476

7. Relation with the pairing between $\mathrm{CH}_{0}(X)$ and $\operatorname{Br}(X)$ 481 References 485

\section{Introduction}

Let $X$ be a smooth, projective, geometrically irreducible variety of dimension $n$ over a field $k$. The conjectural formula of A. A. Beilinson for the conjectural filtration $F \cdot \mathrm{CH}(X)_{\mathbb{Q}}$ of the Chow groups of $X$ reads (see [J3, page 259])

$$
\mathrm{Gr}^{i} \mathrm{CH}^{j}(X)_{\mathbb{Q}} \cong \operatorname{Ext}^{i} \mathscr{M}_{\mathscr{M}_{k}}\left(1, h^{2 j-i}(X)(j)\right) \text {. }
$$

DUKE MATHEMATICAL JOURNAL 
In the particular case of 0-cycles, Poincaré duality allows us to write this in the more simple form

$$
\mathrm{Gr}^{i} \mathrm{CH}_{0}(X)_{\mathbb{Q}} \cong \operatorname{Ext}_{\mathscr{M}}^{i} \mathscr{M}_{k}\left(h^{i}(X), 1\right) .
$$

Conjectures aside, there exist natural maps, in fact, with integral coefficients:

$$
\begin{array}{r}
\mathrm{CH}_{0}(X)=F^{0} \mathrm{CH}_{0}(X) \stackrel{\Delta_{n}^{0}}{\longrightarrow} \operatorname{Ext}_{G_{k}}^{0}\left(H^{0}\left(\bar{X}, \mathbb{G}_{m}\right), \bar{k}^{*}\right), \\
A_{0}(X)=F^{1} \mathrm{CH}_{0}(X) \stackrel{\Delta_{n}^{1}}{\longrightarrow} \operatorname{Ext}_{G_{k}}^{1}\left(H^{1}\left(\bar{X}, \mathbb{G}_{m}\right), \bar{k}^{*}\right) .
\end{array}
$$

The first one of these maps is just the degree map, and the second one, which is a lifting of the Albanese map, is recalled in Section 2. The groups on the right-hand side in (1.3) and (1.4) refer to extensions of discrete $G_{k}$-modules $(\bar{k}$ is an algebraic closure of $k, \bar{X}=X \otimes_{k} \bar{k}$, and $G_{k}$ is the absolute Galois group of $k$ ), a fact that makes these groups bigger than we would like. Namely, if one takes instead, respectively, $\operatorname{Ext}^{0}\left(\mathbb{G}_{m, k}, \mathbb{G}_{m, k}\right)$ and $\operatorname{Ext}^{1}\left(\underline{\mathrm{Pic}}_{X / k}, \mathbb{G}_{m, k}\right)$, extensions as commutative group schemes over $k$, then a similar construction should lead to isomorphisms

$$
\begin{aligned}
\mathrm{Gr}^{0} \mathrm{CH}_{0}(X)_{\mathbb{Q}} \stackrel{\sim}{\longrightarrow} \operatorname{Ext}^{0}\left(\mathbb{G}_{m, k}, \mathbb{G}_{m, k}\right)_{\mathbb{Q}}, \\
\mathrm{Gr}^{1} \mathrm{CH}_{0}(X)_{\mathbb{Q}} \stackrel{\sim}{\longrightarrow} \operatorname{Ext}^{1}\left(\underline{\mathrm{Pic}_{X / k}}, \mathbb{G}_{m, k}\right)_{\mathbb{Q}},
\end{aligned}
$$

reminiscent of (1.2).

In this work we contribute the following.

(a) Based on the paper [J4], we construct in Sections 3 and 4 a natural map, with $T(X) \subset A_{0}(X)$ denoting the Albanese kernel,

$$
T(X)=F^{2} \mathrm{CH}_{0}(X) \stackrel{\Delta_{n}^{2}}{\longrightarrow} \operatorname{Ext}_{G_{k}}^{2}\left(H^{2}\left(\bar{X}, \mathbb{G}_{m}\right), \bar{k}^{*}\right)_{\mathbb{Q}} .
$$

\section{Remark 1.8}

Note that, in contrast with (1.3) and (1.4), we use rational coefficients here. We do not dwell on the question of whether this is strictly necessary or not, but we take advantage of it (see Remark 7.17 for a remark in this respect, due to J.-L. ColliotThélène). Also, we do not make statements here that are comparable to (1.5) and (1.6). On the positive side, however, the construction of $\Delta_{n}^{2}$ is very explicit.

(b) By means of Pontryagin duality, map (1.7) is shown in Section 5 to be equivalent to the set of all $l$-adic higher Abel-Jacobi mappings (see [J3, pages 251-252 and 262263]; see also [R, pages 37-40])

$$
T(X) \stackrel{d_{n}^{2}}{\longrightarrow} H_{\mathrm{cont}}^{2}\left(G_{k}, H^{2 n-2}\left(\bar{X}, \mathbb{Q}_{l}(n)\right)\right),
$$


$l$ prime, $l \neq \operatorname{char}(k)$, provided homological and numerical equivalence coincide for algebraic 1-cycles on $\bar{X}$ (see [K, page 379]) and thus unconditionally for surfaces and for varieties in characteristic zero, among others (see [K, page 380]); see Theorem 5.1 and Remark 5.5.

(c) As one application, we discuss in Section 6 an alternative description of (1.9) for surfaces, along the lines of M. Green in [G], thus relating both points of view (see Corollary 6.15).

(d) Finally, in Section 7 we relate the map (1.7) to the standard pairing (see [M, page 406])

$$
\mathrm{CH}_{0}(X) \otimes_{\mathbb{Z}} \mathrm{Br}(X) \longrightarrow \operatorname{Br}(k)
$$

(see Theorem 7.8).

\section{The map $\Delta_{n}^{1}$}

Let $X$ be as in the introduction. We recall the definition of $\Delta_{n}^{1}$. A reference for this is [CTS]. Let $z$ be a 0 -cycle of degree zero of $X$, and let $Z \subset X$ be a 0 -dimensional closed subset containing the support $|z|$ of $z$. Let $\bar{Z} \subset \bar{X}$ be the 0-dimensional closed subset obtained as the inverse image of $Z$ in $\bar{X}=X \otimes_{k} \bar{k}$. We also denote by $\bar{Z} \subset \bar{X}$ the reduced 0-dimensional subscheme supported by this set. Except for this, as a rule in this paper, we denote by a bar the effect of taking base change from $k$ to the algebraic closure $\bar{k}$ of $k$ or, else, to objects defined directly over $\bar{k}$. We have an exact sequence of discrete $G_{k}$-modules

$$
0 \longrightarrow H^{0}\left(\bar{Z}, \mathbb{G}_{m}\right) / H^{0}\left(\bar{X}, \mathbb{G}_{m}\right) \longrightarrow \operatorname{Pic}(\bar{X}, \bar{Z}) \longrightarrow \operatorname{Pic}(\bar{X}) \longrightarrow 0 .
$$

The group $\operatorname{Pic}(\bar{X}, \bar{Z})$ consists of the isomorphism classes of couples $(\overline{\mathscr{L}}, \bar{\theta})$ with $\overline{\mathscr{L}}$ an invertible sheaf on $\bar{X}$ and $\bar{\theta}: \mathscr{O}_{\bar{Z}} \stackrel{\sim}{\longrightarrow} \overline{\mathscr{L}} \otimes \mathscr{O}_{\bar{Z}}$ an isomorphism. The map $\operatorname{Pic}(\bar{X}, \bar{Z}) \rightarrow \operatorname{Pic}(\bar{X})$ is a forgetful one, sending $[(\overline{\mathscr{L}}, \bar{\theta})]$ to $[\overline{\mathscr{L}}]$. Given $\bar{u} \in H^{0}\left(\bar{Z}, \mathbb{G}_{m}\right)$, the image of $[\bar{u}]$ in $\operatorname{Pic}(\bar{X}, \bar{Z})$ is $\left[\left(\mathscr{O}_{\bar{X}}, \bar{\theta}\right)\right]$ with $\bar{\theta}$ standing now for multiplication by $\bar{u}$. (The notation for $\operatorname{Pic}(\bar{X}, \bar{Z})$ can be traced back, at least, to a paper by H. Gillet and R. Thomason [GT, page 243].)

The exact sequence (2.1) can be deduced alternatively from the cohomology sequence of the (self-defining) exact sequence of sheaves on $\bar{X}$,

$$
0 \longrightarrow \mathbb{G}_{m, \bar{X}, \bar{Z}} \longrightarrow \mathbb{G}_{m, \bar{X}} \longrightarrow \mathbb{G}_{m, \bar{Z}} \longrightarrow 0,
$$

or, which is the same thing, from the long exact sequence obtained from the first hypercohomology spectral sequence of the two-term complex $\mathbb{G}_{m, \bar{X}} \rightarrow \mathbb{G}_{m, \bar{Z}}$.

We note that, unless $k$ is a perfect field, this is different from the sequence obtained from the analogous exact sequence on $X$,

$$
0 \longrightarrow \mathbb{G}_{m, X, Z} \longrightarrow \mathbb{G}_{m, X} \longrightarrow \mathbb{G}_{m, Z} \longrightarrow 0
$$


by taking higher direct images by the structure map $X \rightarrow \operatorname{Spec}(k)$. (Here $Z$ is understood as the reduced 0-dimensional subscheme of $X$ supported by this set.) However, by proper base change (see [Mi1, page 224]), the result is the same, when restricting ourselves to the $l$-primary torsion parts, for primes $l \neq \operatorname{char}(k)$.

The norm map for $Z \rightarrow \operatorname{Spec}(k)$ induces a morphism of $G_{k}$-modules which we also denote as $z$,

$$
H^{0}\left(\bar{Z}, \mathbb{G}_{m}\right) / H^{0}\left(\bar{X}, \mathbb{G}_{m}\right) \stackrel{z}{\longrightarrow} \bar{k}^{*},
$$

and by pushing out (2.1) by (2.3), we obtain a 1-extension representing $\Delta_{n}^{1}([z])$ :

$$
0 \longrightarrow \bar{k}^{*} \longrightarrow \bar{E}_{z} \longrightarrow \operatorname{Pic}(\bar{X}) \longrightarrow 0 .
$$

\section{Remark 2.5}

Note that the result is indeed independent of the particular choice of $Z$. This follows from the functoriality properties in $Z$ of (2.1), (2.2), and (2.3). Actually, the extension $\bar{E}_{z}$ can be described physically as follows. Writing $\bar{z}=\sum_{i=1}^{r} n_{i} \bar{x}_{i}, \sum_{i=1}^{r} n_{i}=0$, the fiber of $\bar{E}_{z}$ above the isomorphism class $[\overline{\mathscr{L}}] \in \operatorname{Pic}(\bar{X})$ of an invertible sheaf $\overline{\mathscr{L}}$ on $\bar{X}$ is given, up to canonical isomorphism, by $\bigotimes_{i=1}^{r} \overline{\mathscr{L}}\left(\bar{x}_{i}\right) \otimes n_{i} \backslash\{\overline{0}\}$.

In order to compare now the map $\Delta_{n}^{1}: A_{0}(X) \rightarrow \operatorname{Ext}_{G_{k}}^{1}\left(\operatorname{Pic}(\bar{X}), \bar{k}^{*}\right)$ with the (integral) $l$-adic Abel-Jacobi map $d_{n}^{1}: A_{0}(X) \rightarrow H_{\text {cont }}^{1}\left(G_{k}, H^{2 n-1}\left(\bar{X}, \mathbb{Z}_{l}(n)\right)\right)$, we consider the restriction map

$$
\operatorname{Ext}_{G_{k}}^{1}\left(\operatorname{Pic}(\bar{X}), \bar{k}^{*}\right) \longrightarrow \operatorname{Ext}_{G_{k}}^{1}\left(\operatorname{Pic}(\bar{X})(l), \bar{k}^{*}\right)
$$

and the isomorphisms

$$
\begin{aligned}
\operatorname{Ext}_{G_{k}}^{1}\left(\operatorname{Pic}(\bar{X})(l), \bar{k}^{*}\right) & \cong \operatorname{Ext}_{G_{k}}^{1}\left(\operatorname{Pic}(\bar{X})(l), \bar{k}^{*}(l)\right) \\
& \cong \operatorname{Ext}_{G_{k}}^{1}\left(H^{1}\left(\bar{X}, \mathbb{Q}_{l} / \mathbb{Z}_{l}(1)\right), \mathbb{Q}_{l} / \mathbb{Z}_{l}(1)\right) \\
& \cong \operatorname{Ext}_{G_{k}}^{1}\left(\mathbb{Z}_{l}, H^{2 n-1}\left(\bar{X}, \mathbb{Z}_{l}(n)\right)\right) \\
& \cong H_{\text {cont }}^{1}\left(G_{k}, H^{2 n-1}\left(\bar{X}, \mathbb{Z}_{l}(n)\right)\right) .
\end{aligned}
$$

The isomorphism (2.7) is induced functorially by the change in the second argument. (In Yoneda-Ext language, to go from the left-hand to the right-hand side, one takes $l$-primary torsion submodules). The isomorphism (2.8) is given by the Kummer exact sequence for $\mathbb{G}_{m, \bar{X}}$. At this point there is obviously no difference between extensions as discrete $G_{k}$-modules and extensions as discrete ( $l$-primary) torsion $G_{k}$-modules. The isomorphism (2.9) is given then by applying Pontryagin duality twisted by (1) (i.e., by taking $\operatorname{Hom}\left(-, \bar{k}^{*}\right)$ ) and using Poincaré duality. The extensions are now taken in the category of continuous $\mathbb{Z}_{l}\left[G_{k}\right]$-modules. The last isomorphism is tautological (see $[\mathrm{J} 1])$. 
PROPOSITION 2.11

The map $d_{n}^{1}$ equals $\Delta_{n}^{1}$ followed by the restriction map (2.6) and the isomorphisms (2.7)-(2.10).

Proof

This follows, by Poincaré duality, from the description of $d_{n}^{1}$ given in [J1] (see also [J2, page 139] or [J4, page 261]). We give the details. For all $r \in \mathbb{N}$, the exact diagram
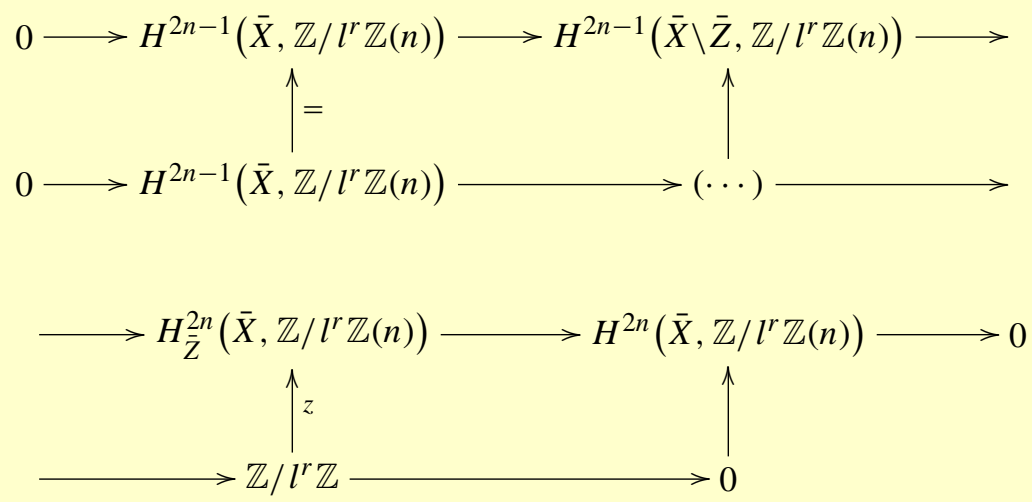

gives, by taking $\operatorname{Hom}\left(-, \bar{\mu}_{l^{r}}\right)$ and using Poincaré duality,

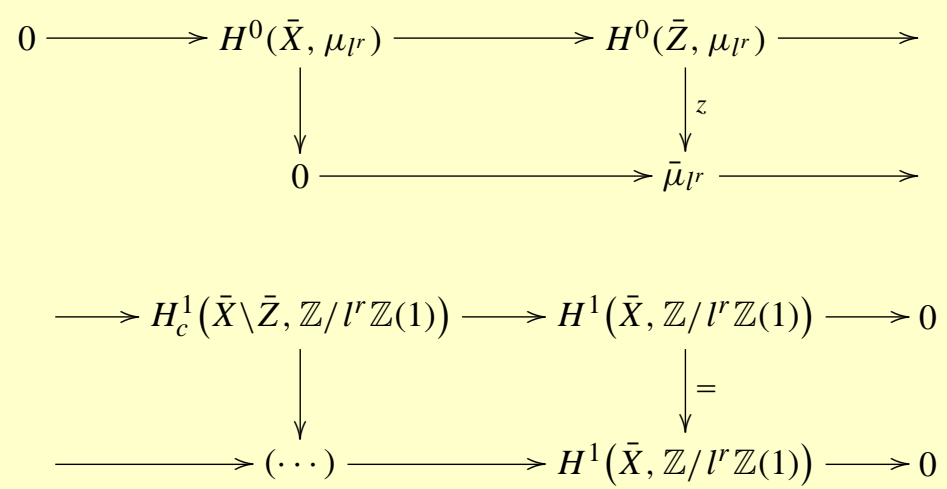

The top row in diagram (2.13) is given by the cohomology sequence of the exact sequence of $l^{r}$-torsion subsheaves in (2.2),

$$
0 \longrightarrow \mu_{l^{r}, \bar{X}, \bar{Z}} \longrightarrow \mu_{l^{r}, \bar{X}} \longrightarrow \mu_{l^{r}, \bar{Z}} \longrightarrow 0
$$

(thus $\mu_{l^{r}, \bar{X}, \bar{Z}}=\bar{j} ! \mu_{l^{r}, \bar{X} \backslash \bar{Z}}$, where the letter $\bar{j}$ denotes the inclusion map $\bar{X} \backslash \bar{Z} \hookrightarrow \bar{X}$ ), whence it is the sequence of $l^{r}$-torsion submodules of the corresponding part of the 
cohomology sequence of (2.2). By the construction of (2.4), it follows that the bottom row in (2.13) equals the sequence of $l^{r}$-torsion submodules in (2.4),

$$
0 \longrightarrow \bar{\mu}_{l^{r}} \longrightarrow{ }^{r} \bar{E}_{z} \longrightarrow{ }_{l^{r}} \operatorname{Pic}(\bar{X}) \longrightarrow 0 .
$$

From this it follows that the image of $\Delta_{n}^{1}([z])$ in $\operatorname{Ext}_{G_{k}}^{1}\left(H^{1}\left(\bar{X}, \mathbb{Q}_{l} / \mathbb{Z}_{l}(1)\right), \mathbb{Q}_{l} / \mathbb{Z}_{l}(1)\right)$ is represented by the inductive limit of these sequences, as $r$ varies. Taking now the Pontryagin dual of this and twisting the result by (1) is tantamount to taking the projective limit of the bottom sequences in (2.12), as $r$ varies. By [J1], [J2, page 139], and [J4, page 261], this is $d_{n}^{1}([z])$.

\section{Construction of $\Delta_{n}^{2}$}

We keep $X$ as before. Let $z$ be a 0 -cycle of $X$ of degree zero such that $\Delta_{n}^{1}([z])=0$. In the notation of Section 2, there exists a morphism of $G_{k}$-modules

$$
\operatorname{Pic}(\bar{X}, \bar{Z}) \stackrel{\tilde{z}}{\longrightarrow} \bar{k}^{*}
$$

restricting to the map $z$ of (2.3) on $H^{0}\left(\bar{Z}, \mathbb{G}_{m}\right) / H^{0}\left(\bar{X}, \mathbb{G}_{m}\right)$. The map $\tilde{z}$ is determined up to a morphism of $G_{k}$-modules $\operatorname{Pic}(\bar{X}) \rightarrow \bar{k}^{*}$.

We now construct, for all 0 -dimensional closed subsets $Z \subset X$ (including the improper case $Z=\emptyset$ ), explicit 2-extensions of discrete $G_{k}$-modules in the style of (2.1),

$$
0 \longrightarrow \operatorname{Pic}(\bar{X}, \bar{Z}) \longrightarrow(\cdots) \longrightarrow(\cdots) \longrightarrow \operatorname{Br}(\bar{X}) \longrightarrow 0,
$$

defining a canonical element $\alpha_{Z} \in \operatorname{Ext}_{G_{k}}^{2}(\operatorname{Br}(\bar{X}), \operatorname{Pic}(\bar{X}, \bar{Z})$ ) (see (3.14) and (3.15)). For $Z_{1} \subset Z_{2}$, we have natural maps of exact sequences

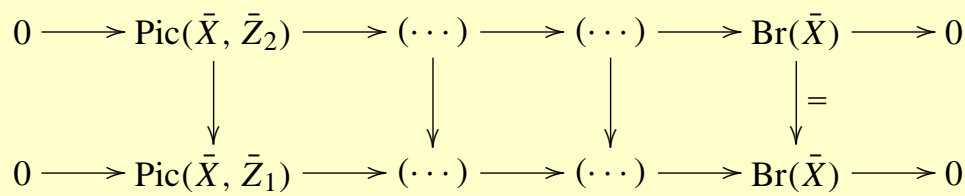

implying that, under the restriction morphism

$$
\operatorname{Ext}_{G_{k}}^{2}\left(\operatorname{Br}(\bar{X}), \operatorname{Pic}\left(\bar{X}, \bar{Z}_{2}\right)\right) \longrightarrow \operatorname{Ext}_{G_{k}}^{2}\left(\operatorname{Br}(\bar{X}), \operatorname{Pic}\left(\bar{X}, \bar{Z}_{1}\right)\right),
$$

$\alpha_{Z_{2}}$ maps to $\alpha_{Z_{1}}$. Moreover, we show that, for $Z=\emptyset, \alpha_{\emptyset}$ is a torsion element in $\operatorname{Ext}_{G_{k}}^{2}(\operatorname{Br}(\bar{X}), \operatorname{Pic}(\bar{X}))$ (see Proposition (3.16)). We then give the following.

\section{Definition 3.4}

Let $z$ be a 0 -cycle of degree zero on $X$, such that $\Delta_{n}^{1}([z])=0$. Let $Z \subset X$ be any 0-dimensional closed subset containing the support $|z|$ of $z$. Then $\Delta_{n}^{2}([z]) \in$ $\operatorname{Ext}_{G_{k}}^{2}\left(\operatorname{Br}(\bar{X}), \bar{k}^{*}\right) \mathbb{Q}$ is the image of $\alpha_{Z}$ under the morphism (3.1), $\Delta_{n}^{2}([z])=\widetilde{z}\left(\alpha_{Z}\right)$. 


\section{Remark 3.5}

(a) It is shown later in Section 3 that the result indeed depends only on the rational equivalence class $[z]$ of $z$ (see Proposition 3.17). So far, we consider this as attached to the cycle $z$ itself, and we write, correspondingly, $\Delta_{n}^{2}(z)$.

(b) For $z$ fixed and $Z$ containing $|z|$, the class $\Delta_{n}^{2}(z)$ does not depend on the chosen $\tilde{z}$ because the variation is measured by a homomorphic image of $\alpha_{\emptyset}$, which is a torsion element and whence is zero when tensored with $\mathbb{Q}$.

(c) For fixed $z$, the result does not depend on $Z$ either, because of the functoriality in $Z$ (see diagram (3.3)).

(d) If $z=z^{\prime}+z^{\prime \prime}$ with $z^{\prime}$ and $z^{\prime \prime} 0$-cycles of degree zero such that $\Delta_{n}^{1}\left(\left[z^{\prime}\right]\right)=0$ and $\Delta_{n}^{1}\left(\left[z^{\prime \prime}\right]\right)=0$, then $\Delta_{n}^{2}(z)=\Delta_{n}^{2}\left(z^{\prime}\right)+\Delta_{n}^{2}\left(z^{\prime \prime}\right)$. To see this, it suffices to take $Z$ so as to contain $\left|z^{\prime}\right| \cup\left|z^{\prime \prime}\right|$ and to choose $\widetilde{z}=\widetilde{z}^{\prime}+\widetilde{z}^{\prime \prime}$.

Definition 3.4 therefore yields a morphism

$$
T(X) \stackrel{\Delta_{n}^{2}}{\longrightarrow} \operatorname{Ext}_{G_{k}}^{2}\left(\operatorname{Br}(\bar{X}), \bar{k}^{*}\right)_{\mathbb{Q}} .
$$

This follows from the inclusion $T(X)_{\mathbb{Q}} \subset \operatorname{Ker}\left(\Delta_{n}^{1}\right)_{\mathbb{Q}}$. We have indeed, more generally, in the notation of Section 2, a commutative diagram

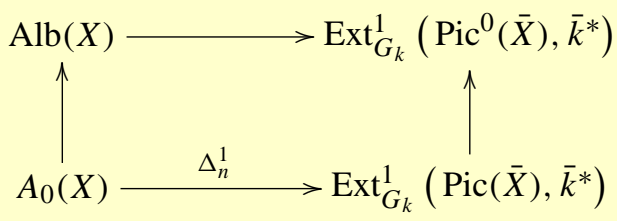

the upper horizontal map coming from the duality between the Albanese variety and the Picard variety. And, second, the right-hand-side vertical map yields an isomorphism when tensored with $\mathbb{Q}$. This follows from the exact sequence

$$
0 \longrightarrow \operatorname{Pic}^{0}(\bar{X}) \longrightarrow \operatorname{Pic}(\bar{X}) \longrightarrow \mathrm{NS}(\bar{X}) \longrightarrow 0
$$

and from the fact that the abelian group $\operatorname{NS}(\bar{X})$ is finitely generated, whence that $\operatorname{Ext}_{G_{k}}^{i}\left(\mathrm{NS}(\bar{X}), \bar{k}^{*}\right)_{\mathbb{Q}}=0$ for $i>0$. We single out this fact since we want to use it again later on.

\section{Remark 3.9}

Let $\bar{M}$ and $\bar{N}$ be discrete $G_{k}$-modules, with $\bar{M}$ finitely generated as an abelian group. Then $\operatorname{Ext}_{G_{k}}^{i}(\bar{M}, \bar{N})_{\mathbb{Q}}=0$ for $i>0$. This follows from [Mi2, Chapter I, Example 0.8] together with [S, Section I.2, Corollaire 3]. 
If $\bar{X}$ is a surface, then $\operatorname{Br}(\bar{X})=H^{2}\left(\bar{X}, \mathbb{G}_{m}\right)$ (see [Mi1, page 149]), and (3.6) is the map announced in (1.7). In the general case we still have an embedding $\operatorname{Br}(\bar{X}) \hookrightarrow$ $H^{2}\left(\bar{X}, \mathbb{G}_{m}\right)$ (see [Mi1, page 142]) which is not known so far to be an isomorphism. In Section 4, we give a cohomological definition for the map (1.7), and it is shown there that it induces the map (3.6) by restriction. This (perhaps temporary) ambiguity in our use of the symbol $\Delta_{n}^{2}$ causes no harm, however, since its precise meaning is always clear from the context.

We now give a construction of (3.2). Because it is very explicit, it may not be pleasant to read. The cohomological description given in Section 4 can be read independently.

\section{Construction of (3.2): Case $Z=\emptyset$}

We treat this case first for the sake of clarity. In the first place, we derive a canonical exact sequence of $G_{k}$-modules

$$
0 \longrightarrow \operatorname{Pic}(\bar{X}) \longrightarrow \prod_{\bar{C} \subset \bar{X}} \operatorname{Pic}(\bar{C}) \longrightarrow \tilde{\operatorname{Br}}(\bar{X}) \longrightarrow \operatorname{Br}(\bar{X}) \longrightarrow 0
$$

which is a prototype for other sequences in this section. Here, in the second term, $\bar{C} \subset \bar{X}$ runs over all closed irreducible curves of $\bar{X}$. This exact sequence is not a sequence of discrete $G_{k}$-modules but of continuous $G_{k}$-modules, when endowed (the two middle terms) with a natural topology stemming from the interpretation of (3.10) as a projective limit of exact sequences of discrete $G_{k}$-modules. We come back to this below.

We view the Brauer group $\operatorname{Br}(Y)$ of a variety $Y$ as the set of equivalence classes of Severi-Brauer varieties over $Y$ (see [Gr1], [Gr2], [Gr3], [Mi1, Chapter III, Section 4, and Chapter IV]). A Severi-Brauer variety $P \rightarrow Y$ (or $P$ for short) of relative dimension $n-1, n \geq 1$, over $Y$ is a proper flat morphism with $\mathbb{P}^{n-1}$ as geometric fibres. Actually, it is a projective morphism. Given such a Severi-Brauer variety, the open subset $\underline{\operatorname{Div}}_{P / Y}^{1}$ of the relative Hilbert scheme $\underline{\text { Hilb }}_{P / Y}$ which parametrizes effective divisors of degree 1 on the fibres of $P \rightarrow Y$ is a Severi-Bauer variety of relative dimension $n-1$ over $Y$ and is called the dual $P^{\curlyvee}$ of $P$. Given Severi-Brauer varieties $P_{i}$ of relative dimensions $n_{i}-1, i=1,2$, the open subset $\operatorname{Div}_{P_{1} \times Y}^{1,1} P_{2} / Y$ of the Hilbert scheme Hilb ${ }_{P_{1} \times Y P_{2} / Y}$ which parametrizes effective divisors of bidegree $(1,1)$ on the fibres of $P_{1} \times_{Y} P_{2} \rightarrow Y$ is a Severi-Brauer variety over $Y$ of relative dimension $n_{1} n_{2}-1$. We denote its dual by $P_{1} * P_{2}$ (see [Gr1, page 64]), and we call this the Segre product of $P_{1}$ and $P_{2}$. There is a canonical embedding $P_{1} \times_{Y} P_{2} \hookrightarrow P_{1} * P_{2}$ over $Y$, which restricts to the Segre embedding on the geometric fibres. Given a SeveriBrauer variety $P$ over $Y$, one has a canonical isomorphism of Severi-Brauer varieties $P * P^{\sim} \stackrel{\sim}{\longrightarrow} \mathbb{P}(\mathscr{A})$ with $\mathscr{A}$ a well-defined locally free sheaf of finite rank on $Y$, actually a $\mathscr{O}_{Y}$-algebra. The Segre product is functorial in $P_{1}$ and $P_{2}$ for isomorphisms, and we 
denote by $f_{1} * f_{2}: P_{1} * P_{2} \stackrel{\sim}{\longrightarrow} P_{1}^{\prime} * P_{2}^{\prime}$ the isomorphism induced by isomorphisms $f_{i}: P_{i} \stackrel{\sim}{\longrightarrow} P_{i}^{\prime}, i=1,2$, of Severi-Brauer varieties.

If $\mathscr{E}_{1}$ and $\mathscr{E}_{2}$ are locally free sheaves of finite rank on $Y$, one has a canonical isomorphism of Severi-Brauer varieties $\mathbb{P}\left(\mathscr{E}_{1}\right) * \mathbb{P}\left(\mathscr{E}_{2}\right) \stackrel{\sim}{\longrightarrow} \mathbb{P}\left(\mathscr{E}_{1} \otimes \mathscr{E}_{2}\right)$, natural in $\mathscr{E}_{1}$ and $\mathscr{E}_{2}$. A Severi-Brauer variety $P \stackrel{\pi}{\rightarrow} Y$ is isomorphic to a projective bundle $\mathbb{P}(\mathscr{E})$ if and only if there exists an invertible sheaf $\Xi$ on $P$, restricting to $\mathscr{O}_{\mathbb{P}^{n-1}}(1)$ on the geometric fibres. Given such a $\Xi$, the canonical epimorphism $\pi^{*}\left(R^{0} \pi_{*} \Xi\right) \rightarrow \Xi$ induces a canonical isomorphism $P \stackrel{\sim}{\longrightarrow} \mathbb{P}\left(R^{0} \pi_{*} \Xi\right)$ and a canonical relative isomorphism $\Xi \stackrel{\sim}{\longrightarrow} \mathscr{O}_{\mathbb{P}\left(R^{0} \pi_{*} \Xi\right)}(1)$. If $P_{i}, i=1,2$, are Severi-Brauer varieties that each admit an invertible sheaf $\Xi_{i}, i=1,2$, satisfying the above property, then we obtain a similar object $\Xi_{1} * \Xi_{2}$ on $P_{1} * P_{2}$ from the canonical isomorphism $P_{1} * P_{2} \stackrel{\sim}{\longrightarrow} \mathbb{P}\left(R^{0} \pi_{1 *} \Xi_{1}\right) * \mathbb{P}\left(R^{0} \pi_{2 *} \Xi_{2}\right) \stackrel{\sim}{\longrightarrow} \mathbb{P}\left(R^{0} \pi_{1 *} \Xi_{1} \otimes R^{0} \pi_{2 *} \Xi_{2}\right)$ by pulling back $\mathscr{O}_{\mathbb{P}\left(R^{0} \pi_{1 *} \Xi_{1} \otimes R^{0} \pi_{2 *} \Xi_{2}\right)}$ (1). If $P$ is a Severi-Brauer variety that admits an invertible sheaf $\Xi$ as above, then one has canonically a similar object $\Xi^{\curlyvee}$ on $P^{\curlyvee}$ and a canonical relative isomorphism $\Xi * \Xi^{\curlyvee} \stackrel{\sim}{\longrightarrow} \mathscr{O}_{\mathbb{P}(\mathscr{A})}(1)$. Namely, from the isomorphism $P \stackrel{\sim}{\longrightarrow} \mathbb{P}\left(R^{0} \pi_{*} \Xi\right)$ we obtain an isomorphism $P^{\vee} \stackrel{\sim}{\longrightarrow} \mathbb{P}\left(R^{0} \pi_{*} \Xi\right)^{*}$, whence a sheaf $\Xi^{\vee}$ by pulling back $\mathscr{O}_{\mathbb{P}}\left(R^{0} \pi_{*} \Xi\right)^{*}(1)$. The rest then follows from the existence of a canonical isomorphism $\mathscr{A} \stackrel{\sim}{\longrightarrow}\left(R^{0} \pi_{*} \Xi\right) \otimes\left(R^{0} \pi_{*} \Xi\right)^{*}$. The passage from $(P, \Xi)$ to $\left(P^{\vee}, \Xi^{\vee}\right)$ is (contravariantly) functorial for isomorphisms.

Two Severi-Brauer varieties $P$ and $P^{\prime}$ over $Y$ are said to be equivalent, $P \sim$ $P^{\prime}$, if and only if there exist locally free sheaves $\mathscr{E}$ and $\mathscr{E}^{\prime}$ of finite rank on $Y$ and an isomorphism over $Y: P * \mathbb{P}(\mathscr{E}) \stackrel{\sim}{\longrightarrow} P^{\prime} * \mathbb{P}\left(\mathscr{E}^{\prime}\right)$. For a Severi-Brauer variety $P$, we denote by $[P]$ its class in the Brauer group $\operatorname{Br}(Y)$. The addition law is given by $\left[P_{1}\right]+\left[P_{2}\right]=\left[P_{1} * P_{2}\right]$. The zero element is represented by $Y=\mathbb{P}\left(\mathscr{O}_{Y}\right)$, and $-[P]=\left[P^{\vee}\right]$. More generally, given $d \in \mathbb{N}$, the open subset $\underline{\operatorname{Div}}_{P / Y}^{d}$ of the relative Hilbert scheme $\underline{\text { Hilb }}_{P / Y}$ which parametrizes effective divisors of degree $d$ on the fibres of $P \rightarrow Y$ is a Severi-Brauer variety over $Y$, representing $-d[P]$. Moreover, one finds that $[P]=0$ if and only if $P$ is isomorphic to a projective bundle $\mathbb{P}(\mathscr{E})$ (see [Gr1, page 64]). It follows that, given $d \in \mathbb{N}$, one has $d[P]=0$ if and only if there exists an invertible sheaf on $P$ restricting to $\mathscr{O}_{\mathbb{P}^{n-1}}(d)$ on the geometric fibres of $P \rightarrow Y$. For example, the existence of the relative canonical sheaf $\omega_{P / Y}$ implies $n[P]=0$. The following fact plays a fundamental role in what follows.

\section{PROPOSITION 3.11}

Let $\bar{C}$ be a curve over an algebraically closed field $\bar{k}$. Then $\operatorname{Br}(\bar{C})=0$. Let $C_{s}$ be a proper curve over a separably closed field $k_{s}$. Then $\operatorname{Br}\left(C_{S}\right)=0$.

\section{Proof (See [Gr3, page 89, Corollaire 1.2, page 132, and Corollaire 5.8])}

We now construct the exact sequence (3.10) from right to left. We have dwelt already 
on the group $\operatorname{Br}(\bar{X})$, and we describe next the group $\widetilde{\operatorname{Br}}(\bar{X})$ and the map onto $\operatorname{Br}(\bar{X})$. Given a Severi-Brauer variety $\bar{P}$ over $\bar{X}$, it follows from Proposition (3.11) that, for all closed irreducible curves $\bar{C} \subset \bar{X}$, there exists an invertible sheaf $\bar{\xi}_{\bar{C}}$ on the restriction $\bar{P}_{\bar{C}}$ of $\bar{P} \rightarrow \bar{X}$ to $\bar{C} \subset \bar{X}$ such that $\bar{\xi}_{\bar{C}}$ restricts to $\mathscr{O}_{\mathbb{P}^{n-1}}(1)$ on the geometric fibres of $\bar{P}_{\bar{C}} \rightarrow \bar{C}$. We define $\widetilde{\operatorname{Br}}(\bar{X})$ to be the set of equivalence classes of couples $(\bar{P}, \bar{\xi})$ with $\bar{P}$ a Severi-Brauer variety over $\bar{X}$ and $\bar{\xi}$ standing for a choice of a $\bar{\xi}_{\bar{C}}$ as above for all $\bar{C}$, modulo the equivalence relation $(\bar{P}, \bar{\xi}) \sim\left(\bar{P}^{\prime}, \bar{\xi}^{\prime}\right)$ if and only if there exist locally free sheaves $\overline{\mathscr{E}}$ and $\overline{\mathscr{E} \prime}$ of finite rank on $\bar{X}$, an isomorphism $\bar{P} * \mathbb{P}(\overline{\mathscr{E}}) \stackrel{\sim}{\longrightarrow}$ $\bar{P}^{\prime} * \mathbb{P}\left(\overline{\mathscr{E}}^{\prime}\right)$ over $\bar{X}$, and a relative isomorphism $\bar{\xi} * \mathscr{O}_{\mathbb{P}(\overline{\mathscr{E}})}(1) \stackrel{\sim}{\longrightarrow} \bar{\xi}^{\prime} * \mathscr{O}_{\mathbb{P}(\overline{\mathscr{E}})}(1)$. By this we mean, for all $\bar{C}$, a relative isomorphism $\bar{\xi}_{\bar{C}} * \mathscr{O}_{\mathbb{P}\left(\overline{\mathscr{E}}_{\bar{C}}\right)}(1) \stackrel{\sim}{\longrightarrow} \bar{\xi}_{\bar{C}}^{\prime} * \mathscr{O}_{\mathbb{P}\left(\overline{\mathscr{E}}_{\bar{C}}^{\prime}\right)}(1)$ over the induced isomorphism $\bar{P}_{\bar{C}} * \mathbb{P}\left(\overline{\mathscr{E}}_{\bar{C}}\right) \stackrel{\sim}{\longrightarrow} \bar{P}_{\bar{C}}^{\prime} * \mathbb{P}\left(\overline{\mathscr{E}}_{\bar{C}}^{\prime}\right)$. It is straightforward to check that this is indeed an equivalence relation. We write $[(\bar{P}, \bar{\xi})]$ for the equivalence class of $(\bar{P}, \bar{\xi})$. Moreover, we define $\left(\bar{P}_{1}, \bar{\xi}_{1}\right) *\left(\bar{P}_{2}, \bar{\xi}_{2}\right)=\left(\bar{P}_{1} * \bar{P}_{2}, \bar{\xi}_{1} * \bar{\xi}_{2}\right)$ with $\left(\bar{\xi}_{1} * \bar{\xi}_{2}\right)_{\bar{C}}=\bar{\xi}_{1, \bar{C}} * \bar{\xi}_{2, \bar{C}}$ for all $\bar{C} \subset \bar{X}$. This induces an addition law in the quotient set $\operatorname{Br}(\bar{X})$. The zero element is represented by $(\bar{X}, \overline{\mathscr{O}})$ with $\overline{\mathscr{O}}_{\bar{C}}=\mathscr{O}_{\bar{C}}$ for all $\bar{C}$. Also, $-[(\bar{P}, \bar{\xi})]=\left[\left(\bar{P}^{\vee}, \bar{\xi}^{\vee}\right)\right]$ with $\bar{\xi}_{\bar{C}}^{\vee}=\left(\bar{\xi}_{\bar{C}}\right)^{\vee}$ for all $\bar{C}$. The surjection $\widetilde{\operatorname{Br}}(\bar{X}) \rightarrow \operatorname{Br}(\bar{X})$ is the forgetful map sending $[(\bar{P}, \bar{\xi})]$ to $[\bar{P}]$.

The map $\prod_{\bar{C} \subset \bar{X}} \operatorname{Pic}(\bar{C}) \rightarrow \widetilde{\operatorname{Br}}(\bar{X})$ sends $\left(\left[\overline{\mathscr{L}}_{\bar{C}}\right]\right)_{\bar{C} \subset \bar{X}}$ to $[(\bar{X}, \bar{\lambda})]$ with $\bar{\lambda}_{\bar{C}}=\overline{\mathscr{L}}_{\bar{C}}$ for all $\bar{C} \subset \bar{X}$. This element belongs to the kernel of $\widetilde{\operatorname{Br}}(\bar{X}) \rightarrow \operatorname{Br}(\bar{X})$. Conversely, given $[(\bar{P}, \bar{\xi})] \in \widetilde{\operatorname{Br}}(\bar{X})$ such that $[\bar{P}]=0$ in $\operatorname{Br}(\bar{X})$, there exist locally free sheaves $\overline{\mathscr{E}}, \overline{\mathscr{E} \prime}$ of finite rank on $\bar{X}$ and an isomorphism of Severi-Brauer varieties $\bar{P} * \mathbb{P}(\overline{\mathscr{E}}) \stackrel{\sim}{\longrightarrow}$ $\bar{X} * \mathbb{P}\left(\overline{\mathscr{E}}^{\prime}\right)=\mathbb{P}\left(\overline{\mathscr{E}}^{\prime}\right)$. By the seesaw principle, this implies the existence of a relative isomorphism $\bar{\xi} * \mathscr{O}_{\mathbb{P}(\overline{\mathscr{E}})}(1) \stackrel{\sim}{\longrightarrow} \bar{\lambda} * \mathscr{O}_{\mathbb{P}(\overline{\mathscr{E}})}(1)$ for a suitable $\bar{\lambda}$ as above, and hence $(\bar{P}, \bar{\xi}) \sim(\bar{X}, \bar{\lambda})$; that is, $[(\bar{P}, \bar{\xi})]$ lies in the image of the above map.

The map $\operatorname{Pic}(\bar{X}) \rightarrow \prod_{\bar{C} \subset \bar{X}} \operatorname{Pic}(\bar{C})$ sends $[\overline{\mathscr{L}}]$ to $\left(\left[\overline{\mathscr{L}} \otimes \mathscr{O}_{\bar{C}}\right]\right) \bar{C} \subset \bar{X}$. The image of this map is the kernel of the preceding one. Namely, given $\left(\left[\overline{\mathscr{L}}_{\bar{C}}\right]\right)_{\bar{C}} \subset \bar{X}$ as above, one has $(\bar{X}, \bar{\lambda}) \sim(\bar{X}, \overline{\mathscr{O}})$ if and only if there exist locally free sheaves $\overline{\mathscr{E}}$ and $\overline{\mathscr{E}}^{\prime}$ of finite rank on $\bar{X}$, an isomorphism $\mathbb{P}(\overline{\mathscr{E}}) \stackrel{\sim}{\longrightarrow} \mathbb{P}\left(\overline{\mathscr{E}}^{\prime}\right)$, and a relative isomorphism $\bar{\lambda} * \mathscr{O}_{\mathbb{P}(\overline{\mathscr{E}})}(1) \stackrel{\sim}{\longrightarrow} \mathscr{O}_{\mathbb{P}(\overline{\mathscr{E}})}(1)$. If this is given, then we deduce, again by seesaw, a relative isomorphism $\overline{\mathscr{L}} \otimes \mathscr{O}_{\mathbb{P}(\overline{\mathscr{E}})}(1) \stackrel{\sim}{\longrightarrow} \mathscr{O}_{\mathbb{P}\left(\overline{\mathscr{E}}^{\prime}\right)}(1)$ for a suitable invertible sheaf $\overline{\mathscr{L}}$ on $\bar{X}$, whence $\bar{\lambda}_{\bar{C}} \stackrel{\sim}{\longrightarrow} \overline{\mathscr{L}} \otimes \mathscr{O}_{\bar{C}}$ for all $\bar{C} \subset \bar{X}$. Conversely, if this occurs, then the preceding condition is satisfied for $\overline{\mathscr{E}}=\mathscr{O}_{\bar{X}}$ and $\overline{\mathscr{E}}^{\prime}=\overline{\mathscr{L}}$.

It remains to be shown that the map $\operatorname{Pic}(\bar{X}) \rightarrow \prod_{\bar{C} \subset \bar{X}} \operatorname{Pic}(\bar{C})$ is injective. By repeated application of [SGA2, Exposé XII, Corollaire 3.6], we reduce this to the case where $\bar{X}$ is a surface. Then let $\overline{\mathscr{L}}$ be an invertible sheaf on $\bar{X}$ such that $\mathscr{O}_{\bar{C}} \cong \overline{\mathscr{L}} \otimes \mathscr{O}_{\bar{C}}$ for all closed irreducible curves $\bar{C} \subset \bar{X}$. Consider a Lefschetz pencil on $\bar{X}$, and let $\bar{g}: \bar{X}^{\prime} \rightarrow \mathbb{P}_{\bar{k}}^{1}$ be the morphism obtained by blowing up the basis locus. It suffices to show that the pullback $\overline{\mathscr{L}}^{\prime}$ of $\overline{\mathscr{L}}$ to $\bar{X}^{\prime}$ is trivial. We have $\bar{g}^{*}\left(R^{0} \bar{g}_{*} \overline{\mathscr{L}}^{\prime}\right) \cong \overline{\mathscr{L}}^{\prime}$ and 
$R^{0} \bar{g}_{*} \overline{\mathscr{L}}^{\prime} \cong \mathscr{O}_{\mathbb{P}_{\bar{k}}^{1}}$ since this is now (relatively) isomorphic to the restriction of $\overline{\mathscr{L}}^{\prime}$ to any of the exceptional divisors. Hence the result follows.

We also need a more refined version of this, to the effect that we may choose a finite set of closed irreducible curves $\bar{C}_{1}, \ldots, \bar{C}_{r}$ on $\bar{X}$ such that the restriction map $\operatorname{Pic}(\bar{X}) \rightarrow \prod_{i=1}^{r} \operatorname{Pic}\left(\bar{C}_{i}\right)$ is injective. To this end, we choose first a finite set of such curves such that their classes generate the group $\operatorname{Num}_{1}(\bar{X})$ of algebraic 1-cycles on $\bar{X}$ modulo numerical equivalence. Write $N=\mid$ Tors $\mathrm{NS}(\bar{X}) \mid$. If an invertible sheaf $\overline{\mathscr{L}}$ on $\bar{X}$ restricts to the trivial sheaf on each of these curves, then $\left[\overline{\mathscr{L}}^{\otimes N}\right]$ belongs to $\operatorname{Pic}^{0}(\bar{X}) \subset \operatorname{Pic}(\bar{X})$. Consider then a smooth irreducible curve $\bar{C}$, an iterated hyperplane section of $\bar{X}$. By weak Lefschetz, the restriction morphism $\operatorname{Pic}^{0}(\bar{X}) \rightarrow \operatorname{Pic}^{0}(\bar{C})$ has a finite kernel (of $p$-torsion if $\operatorname{char}(k)=p>0$ and trivial otherwise). Adding this curve to the previous list yields, therefore, a restriction map with a finite kernel. Adding then for each nontrivial element in this kernel a curve where this element has a nontrivial restriction, the claimed list is complete.

Let $\bar{S}$ be a finite set of closed irreducible curves of $\bar{X}$ such that

(1) $\bar{S}$ is closed under conjugation by $G_{k}$ and

(2) $\operatorname{Pic}(\bar{X}) \hookrightarrow \prod_{\bar{C} \in \bar{S}} \operatorname{Pic}(\bar{C})$ via the restriction map.

Then the construction of (3.10) may be repeated verbatim, replacing the set of all closed irreducible curves $\bar{C} \subset \bar{X}$ by this set $\bar{S}$ and obtaining an exact sequence of discrete $G_{k}$-modules

$$
0 \longrightarrow \operatorname{Pic}(\bar{X}) \longrightarrow \prod_{\bar{C} \in \bar{S}} \operatorname{Pic}(\bar{C}) \longrightarrow \tilde{\operatorname{Br}}_{\bar{S}}(\bar{X}) \longrightarrow \operatorname{Br}(\bar{X}) \longrightarrow 0 .
$$

Moreover, if $\bar{S}_{1} \subset \bar{S}_{2}$, then the sequence (3.12) for $\bar{S}_{2}$ maps onto the sequence for $\bar{S}_{1}$, with fixed ends. Thus all these sequences represent one and the same element $\alpha_{\emptyset} \in \operatorname{Ext}_{G_{k}}^{2}(\operatorname{Br}(\bar{X}), \operatorname{Pic}(\bar{X}))$. As $\bar{S}$ varies, the sequences (3.12) form a projective system, and the projective limit is (3.10).

\section{Construction of (3.2): The general case}

We build on (3.10). Endowing everything with a trivialization above the points of $\bar{Z}$ and taking this trivialization into account in the definition of the corresponding equivalence relations, we construct first a sequence of (nondiscrete) $G_{k}$-modules

$$
0 \longrightarrow \operatorname{Pic}(\bar{X}, \bar{Z}) \longrightarrow \prod_{\bar{C} \subset \bar{X}} \operatorname{Pic}(\bar{C}, \bar{Z} \cap \bar{C}) \longrightarrow \tilde{\operatorname{Br}}(\bar{X}, \bar{Z}) \longrightarrow \operatorname{Br}(\bar{X}) \longrightarrow 0 .
$$

(The last term actually should read $\operatorname{Br}(\bar{X}, \bar{Z})$, but this coincides with $\operatorname{Br}(\bar{X})$.) In the second term, the product is taken over all closed irreducible curves $\bar{C} \subset \bar{X}$.

To define $\widetilde{\operatorname{Br}}(\bar{X}, \bar{Z})$, we consider 4-tuples $(\bar{P}, \bar{\xi}, \bar{f}, \bar{\varphi})$ described as follows. The first two items are as before: $\bar{P} \rightarrow \bar{X}$ is a Severi-Brauer variety, say, of relative 
dimension $n-1$, and $\bar{\xi}$ symbolizes a choice of an invertible sheaf $\bar{\xi}_{\bar{C}}$ on $\bar{P}_{\bar{C}}$ for all $\bar{C} \subset \bar{X}$, restricting to $\mathscr{O}(1)$ on the geometric fibres of $\bar{P}_{\bar{C}} \rightarrow \bar{C}$. Moreover, $\bar{f}$ : $\mathbb{P}_{\bar{Z}}^{n-1} \stackrel{\sim}{\longrightarrow} \bar{P}_{\bar{Z}}$ is an isomorphism, and $\bar{\varphi}$ stands for a selection, for all $\bar{C} \subset \bar{X}$, of a relative isomorphism $\bar{\varphi}_{\bar{C}}: \mathscr{O}_{\mathbb{P}_{\bar{Z} \cap \bar{C}}^{n-1}}(1) \stackrel{\sim}{\longrightarrow} \bar{\xi}_{\bar{C}} \otimes \mathscr{O}_{\bar{P}_{\bar{Z} \cap \bar{C}}}$ over the restriction of $\bar{f}$ above $\bar{Z} \cap \bar{C}$. One defines an equivalence relation for these 4-tuples by setting $(\bar{P}, \bar{\xi}, \bar{f}, \bar{\varphi}) \sim$ $\left(\bar{P}^{\prime}, \bar{\xi}^{\prime}, \bar{f}^{\prime}, \bar{\varphi}^{\prime}\right)$ if and only if there exist locally free sheaves $\overline{\mathscr{E}}$ and $\overline{\mathscr{E}}^{\prime}$ of finite rank on $\bar{X}$ and an isomorphism $\bar{g}: \bar{P} * \mathbb{P}(\overline{\mathscr{E}}) \stackrel{\sim}{\longrightarrow} \bar{P}^{\prime} * \mathbb{P}\left(\overline{\mathscr{E}}^{\prime}\right)$ over $\bar{X}$ and a relative isomorphism $\bar{\psi}: \bar{\xi} * \mathscr{O}_{\mathbb{P}(\overline{\mathscr{E}})}(1) \stackrel{\sim}{\longrightarrow} \bar{\xi}^{\prime} * \mathscr{O}_{\mathbb{P}\left(\overline{\mathscr{E}}^{\prime}\right)}(1)$ such that the composite relative isomorphisms

$$
\begin{aligned}
& \left(\bar{\xi}_{\bar{C}} \otimes \mathscr{O}_{\bar{P}_{\bar{Z} \cap \bar{C}}}\right) * \mathscr{O}_{\mathbb{P}\left(\overline{\mathscr{E}}_{\bar{Z} \cap \bar{C}}\right)}(1) \stackrel{\bar{\psi}_{\bar{C}}}{\longrightarrow}\left(\bar{\xi}_{\bar{C}}^{\prime} \otimes \mathscr{O}_{\bar{P}_{\bar{Z} \cap \bar{C}}}\right) * \mathscr{O}_{\mathbb{P}\left(\overline{\mathscr{E}}_{\bar{Z} \cap \bar{C}}^{\prime}\right)} \\
& \uparrow \bar{\varphi}_{\bar{C}} * 1 \\
& \mathscr{O}_{\mathbb{P}_{\bar{Z} \cap \bar{C}}^{n-1}}(1) * \mathscr{O}_{\mathbb{P}\left(\overline{\mathscr{E}}_{\overline{\mathrm{Z}} \cap \bar{C}}\right)} \\
& \begin{aligned}
\downarrow\left(\bar{\varphi}_{\bar{C}}^{\prime} * 1\right)^{-1} \\
\mathscr{O}_{\mathbb{P}_{\bar{Z} \cap \bar{C}}^{n^{\prime}-1}(1) * \mathscr{O}_{\mathbb{P}(\overline{\mathscr{E}},}(\overline{\bar{Z}} \cap \bar{C})}(1)
\end{aligned}
\end{aligned}
$$

are independent from $\bar{C}$ at every point of $\bar{Z} \cap \bar{C}$. The verification of this fact (and similar ones below) is a straightforward exercise. The set of equivalence classes thus obtained is $\widetilde{\operatorname{Br}}(\bar{X}, \bar{Z})$, and we write $[(\bar{P}, \bar{\xi}, \bar{f}, \bar{\varphi})]$ for the class of $(\bar{P}, \bar{\xi}, \bar{f}, \bar{\varphi})$. An addition law is defined in $\widetilde{\operatorname{Br}}(\bar{X}, \bar{Z})$ by setting $\left[\left(\bar{P}_{1}, \bar{\xi}_{1}, \bar{f}_{1}, \bar{\varphi}_{1}\right)\right]+$ $\left[\left(\bar{P}_{2}, \bar{\xi}_{2}, \bar{f}_{2}, \bar{\varphi}_{2}\right)\right]=\left[\left(\bar{P}_{1} * \bar{P}_{2}, \bar{\xi}_{1} * \bar{\xi}_{2}, \bar{f}_{1} * \bar{f}_{2}, \bar{\varphi}_{1} * \bar{\varphi}_{2}\right)\right]$. (This bears implicitly the choice of an (arbitrary) isomorphism $\mathbb{P}_{\bar{Z}}^{n_{1}-1} * \mathbb{P}_{\bar{Z}}^{n_{2}-1} \stackrel{\sim}{\longrightarrow} \mathbb{P}_{\bar{Z}}^{n_{1} n_{2}-1}$ and a relative isomorphism $\mathscr{O}_{\mathbb{P}_{\bar{Z}}^{n_{1}-1}}(1) * \mathscr{O}_{\mathbb{P}_{\bar{Z}}^{n_{2}-1}}(1) \stackrel{\sim}{\longrightarrow} \mathscr{O}_{\mathbb{P}_{\bar{Z}}^{n_{1} n_{2}-1}}(1)$.) The zero element is represented by $(\bar{X}, \overline{\mathscr{O}}, 1,1)$, where the two last symbols stand for the canonical identifications $\mathbb{P}_{\bar{Z}}^{0} \stackrel{\sim}{\longrightarrow} \bar{Z}$ and $O_{\mathbb{P}_{\bar{Z} \cap \bar{C}}^{0}}(1) \stackrel{\sim}{\longrightarrow} O_{\bar{Z} \cap \bar{C}}$ for all $\bar{C}$. Furthermore, one checks that $-[(\bar{P}, \bar{\xi}, \bar{f}, \bar{\varphi})]=\left[\left(\bar{P}^{`}, \bar{\xi}^{\swarrow},\left(\bar{f}^{`}\right)^{-1},\left(\bar{\varphi}^{`}\right)^{-1}\right)\right]$. The epimorphism $\widetilde{\operatorname{Br}}(\bar{X}, \bar{Z}) \rightarrow \operatorname{Br}(\bar{X})$ is the forgetful map, sending $[(\bar{P}, \bar{\xi}, \bar{f}, \bar{\varphi})]$ to $[\bar{P}]$.

The map $\prod_{\bar{C} \subset \bar{X}} \operatorname{Pic}(\bar{C}, \bar{Z} \cap \bar{C}) \rightarrow \widetilde{\operatorname{Br}}(\bar{X}, \bar{Z})$ sends $\left(\left[\overline{\mathscr{L}}_{\bar{C}}, \bar{\theta}_{\bar{C}}\right]\right)_{\bar{C} \subset \bar{X}}$ to $[(\bar{X}, \bar{\lambda}, 1, \bar{\vartheta})]$, where $\bar{\lambda}_{\bar{C}}=\overline{\mathscr{L}}_{\bar{C}}$ for all $\bar{C} \subset \bar{X}$ and $\bar{\vartheta}$ stands for the collection $\left(\bar{\theta}_{\bar{C}}\right)_{\bar{C} \subset \bar{X}}$. This element obviously belongs to the kernel of $\widetilde{\operatorname{Br}}(\bar{X}, \bar{Z}) \rightarrow \operatorname{Br}(\bar{X})$. Conversely, given $[(\bar{P}, \bar{\xi}, \bar{f}, \bar{\varphi})] \in \widetilde{\operatorname{Br}}(\bar{X}, \bar{Z})$ such that $[\bar{P}]=0$ in $\operatorname{Br}(\bar{X})$, we have seen already that there exist locally free sheaves $\overline{\mathscr{E}}$ and $\overline{\mathscr{E}}^{\prime}$ of finite rank on $\bar{X}$, an isomorphism $\bar{g}: \bar{P} * \mathbb{P}(\overline{\mathscr{E}}) \stackrel{\sim}{\longrightarrow} \mathbb{P}\left(\overline{\mathscr{E}}^{\prime}\right)$, and, for a suitable $\bar{\lambda}$ as before, a relative isomorphism $\bar{\psi}: \bar{\xi} * \mathscr{O}_{\mathbb{P}(\overline{\mathscr{E}})}(1) \stackrel{\sim}{\longrightarrow} \bar{\lambda} * \mathscr{O}_{\mathbb{P}\left(\overline{\mathscr{E}}^{\prime}\right)}(1)$. We exhibit a $\bar{\vartheta}$ as above such that $(\bar{P}, \bar{\xi}, \bar{f}, \bar{\varphi}) \sim(\bar{X}, \bar{\lambda}, 1, \bar{\vartheta})$, thereby showing that the chosen element belongs to the image of our map. To this end, we consider the composite isomorphism

$$
\mathbb{P}_{\bar{Z}}^{n-1} * \mathbb{P}\left(\overline{\mathscr{E}}_{\bar{Z}}\right) \stackrel{\bar{f} * 1}{\longrightarrow} \bar{P}_{\bar{Z}} * \mathbb{P}\left(\overline{\mathscr{E}}_{\bar{Z}}\right) \stackrel{\bar{g}}{\longrightarrow} \mathbb{P}\left(\overline{\mathscr{E}}_{\bar{Z}}^{\prime}\right)
$$

and choose an arbitrary relative isomorphism $\bar{\gamma}: \mathscr{O}_{\mathbb{P}_{\bar{Z}}^{n-1}}(1) * \mathscr{O}_{\mathbb{P}\left(\overline{\mathscr{E}}_{\bar{Z}}\right)}(1) \stackrel{\sim}{\longrightarrow} \mathscr{O}_{\mathbb{P}\left(\overline{\mathscr{E}}_{\bar{Z}}^{\prime}\right)}(1)$. 
Then the composition of relative isomorphisms

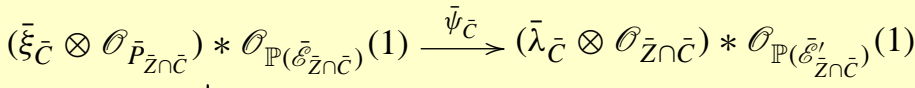

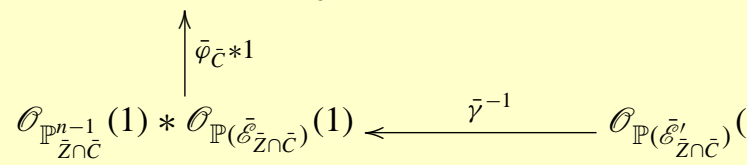

is an isomorphism of $\mathscr{O}_{\mathbb{P}\left(\overline{\mathscr{E}}_{\bar{Z} \cap}^{\prime} \cap \bar{C}\right)}$-modules, and therefore it determines an isomorphism $\bar{\theta}_{\bar{C}}: \mathscr{O}_{\bar{Z} \cap \bar{C}} \stackrel{\sim}{\longrightarrow} \bar{\lambda}_{\bar{C}} \otimes \mathscr{O}_{\bar{Z} \cap \bar{C}}$. By its very construction, $\bar{\vartheta}=\left(\bar{\theta}_{\bar{C}}\right)_{\bar{C} \subset \bar{X}}$ yields the claimed equivalence.

The map $\operatorname{Pic}(\bar{X}, \bar{Z}) \rightarrow \prod_{\bar{C} \subset \bar{X}} \operatorname{Pic}(\bar{C}, \bar{Z} \cap \bar{C})$ sends $[(\overline{\mathscr{L}}, \bar{\theta})]$ to $\left(\left[\left(\overline{\mathscr{L}}_{\otimes} \otimes \mathscr{O}_{\bar{C}}, \bar{\theta} \mid \bar{Z} \cap\right.\right.\right.$ $\bar{C})])_{\bar{C}} \bar{X}$. The image of the latter element in $\widetilde{B r}(\bar{X}, \bar{Z})$ is represented by $(\bar{X}, \bar{\lambda}, 1, \bar{\vartheta})$ with $\bar{\lambda}_{\bar{C}}=\overline{\mathscr{L}} \otimes \mathscr{O}_{\bar{C}}$ for all $\bar{C} \subset \bar{X}$, and $\bar{\vartheta}$ standing for the collection $(\bar{\theta} \mid \bar{Z} \cap \bar{C})_{\bar{C} \subset \bar{X}}$. Now one finds $(\bar{X}, \bar{\lambda}, 1, \bar{\vartheta}) \sim(\bar{X}, \overline{\mathscr{O}}, 1,1)$ by taking $\overline{\mathscr{E}}=\mathscr{O}_{\bar{X}}, \overline{\mathscr{E}}^{\prime}=\overline{\mathscr{L}}, \bar{g}=1_{\bar{X}}$, and $\bar{\psi}=\mathrm{id}: \overline{\mathscr{L}} \rightarrow \mathscr{O}_{\mathbb{P}(\overline{\mathscr{L}})}(1)$. Thus the image of the above map is contained in the kernel of the preceding one. We show the opposite inclusion. In the notation of the preceding paragraph, let $\left(\left[\overline{\mathscr{L}}_{\bar{C}}, \bar{\theta}_{\bar{C}}\right]\right)_{\bar{C} \subset \bar{X}}$ be such that $(\bar{X}, \bar{\lambda}, 1, \bar{\vartheta}) \sim(\bar{X}, \overline{\mathscr{O}}, 1,1)$. Then, as we have already seen, there exists an invertible sheaf $\overline{\mathscr{L}}$ on $\bar{X}$ and isomorphisms $\bar{\beta}_{\bar{C}}$ : $\overline{\mathscr{L}} \otimes \mathscr{O}_{\bar{C}} \stackrel{\sim}{\longrightarrow} \overline{\mathscr{L}}_{\bar{C}}$ for all $\bar{C} \subset \bar{X}$. These are such that the isomorphisms $\left(\bar{\beta}_{\bar{C}} \mid \bar{Z} \cap\right.$ $\bar{C})^{-1} \circ \bar{\theta}_{\bar{C}}: \mathscr{O}_{\bar{Z} \cap \bar{C}} \stackrel{\sim}{\longrightarrow} \overline{\mathscr{L}} \otimes \mathscr{O}_{\bar{Z} \cap \bar{C}}$ are independent from $\bar{C}$ at the points of $\bar{Z} \cap \bar{C}$, whence they define an isomorphism $\bar{\theta}: \mathscr{O}_{\bar{Z}} \stackrel{\sim}{\longrightarrow} \overline{\mathscr{L}} \otimes \mathscr{O}_{\bar{Z}}$, and hence $\left(\left[\left(\overline{\mathscr{L}}_{\bar{C}}, \bar{\theta}_{\bar{C}}\right)\right]\right) \bar{C} \subset \bar{X}$ is the image of $[(\overline{\mathscr{L}}, \bar{\theta})] \in \operatorname{Pic}(\bar{X}, \bar{Z})$.

Finally, we show the injectivity of $\operatorname{Pic}(\bar{X}, \bar{Z}) \rightarrow \prod_{\bar{C} \subset X} \operatorname{Pic}(\bar{C}, \bar{Z} \cap \bar{C})$. Given $[(\overline{\mathscr{L}}, \bar{\theta})]$ mapping to zero, we know already that $\overline{\mathscr{L}} \cong \mathscr{O}_{\bar{X}}$, and we may assume that, actually, $\overline{\mathscr{L}}=\mathscr{O}_{\bar{X}}$. Then $\bar{\theta} \in H^{0}\left(\bar{Z}, \mathscr{O}_{\bar{Z}}^{*}\right)$, and we have to show that $\bar{\theta} \in H^{0}\left(\bar{X}, \mathscr{O}_{\bar{X}}^{*}\right)=\bar{k}^{*}$. Let $\bar{C} \subset \bar{X}$ be a closed irreducible curve containing $\bar{Z}$. Then, by hypothesis, $\bar{\theta} \in H^{0}\left(\bar{C}, \mathscr{O}_{\bar{C}}^{*}\right)=\bar{k}^{*}$, and the result follows.

Let $\bar{S}$ be a finite set of closed irreducible curves of $\bar{X}$ such that

(1) $\bar{S}$ is closed under conjugation by $G_{k}$,

(2) $\operatorname{Pic}(\bar{X}) \hookrightarrow \prod_{\bar{C} \in \bar{S}} \operatorname{Pic}(\bar{C})$ via the restriction maps, and

(3) any two points of $\bar{Z}$ can be connected by a chain of subsets $\bar{Z} \cap \bar{C}, \bar{C} \in \bar{S}$.

(We say $\bar{S}$ is adequate for $Z$.)

Then the construction of (3.13) can be repeated as it stands, replacing the set of all closed curves $\bar{C} \subset \bar{X}$ by $\bar{S}$, and one obtains an exact sequence of discrete $G_{k}$-modules

$$
0 \longrightarrow \operatorname{Pic}(\bar{X}, \bar{Z}) \longrightarrow \prod_{\bar{C} \in \bar{S}} \operatorname{Pic}(\bar{C}, \bar{Z} \cap \bar{C}) \longrightarrow \tilde{\operatorname{Br}}_{\bar{S}}(\bar{X}, \bar{Z}) \longrightarrow \operatorname{Br}(\bar{X}) \longrightarrow 0
$$


(Assumptions (2) and (3) are made to ensure exactness at the term $\operatorname{Pic}(\bar{X}, \bar{Z})$.) Moreover, if $\bar{S}_{1} \subset \bar{S}_{2}$, then we have a natural map of sequences from (3.14) for $\bar{S}_{2}$ to (3.14) for $\bar{S}_{1}$. Thus all these extensions represent the same element

$$
\alpha_{Z} \in \operatorname{Ext}_{G_{k}}^{2}(\operatorname{Br}(\bar{X}), \operatorname{Pic}(\bar{X}, \bar{Z})) .
$$

As $\bar{S}$ varies, the sequences (3.12) form a projective system, and the projective limit is (3.13).

If $Z_{1} \subset Z_{2}$, then one has a natural restriction map from the sequence (3.13) for $Z_{2}$ to the sequence (3.13) for $Z_{1}$. By choosing $\bar{S}$ adequate for both of $Z_{1}$ and $Z_{2}$, we obtain a similar map from the sequence (3.14) for $Z_{2}$ to the sequence (3.14) for $Z_{1}$. This fulfills the announcement made about (3.3), and shows that the morphism

$$
\operatorname{Ext}_{G_{k}}^{2}\left(\operatorname{Br}(\bar{X}), \operatorname{Pic}\left(\bar{X}, \bar{Z}_{2}\right)\right) \longrightarrow \operatorname{Ext}_{G_{k}}^{2}\left(\operatorname{Br}(\bar{X}), \operatorname{Pic}\left(\bar{X}, \bar{Z}_{1}\right)\right)
$$

induced by the restriction map $\operatorname{Pic}\left(\bar{X}, \bar{Z}_{2}\right) \rightarrow \operatorname{Pic}\left(\bar{X}, \bar{Z}_{1}\right)$ sends $\alpha_{Z_{2}}$ to $\alpha_{Z_{1}}$.

We show next that the class $\alpha_{\emptyset}$ is a torsion element.

PROPOSITION 3.16

Let $X$ be a smooth, projective, geometrically irreducible variety over a field $k$. In the notation introduced at the beginning of Section 3, one has $\alpha_{\emptyset}=0$ in $\operatorname{Ext}_{G_{k}}^{2}(\operatorname{Br}(\bar{X}), \operatorname{Pic}(\bar{X}))_{\mathbb{Q}}$.

\section{Proof}

We choose a finite set $\bar{S}$ of closed irreducible curves $\bar{C} \subset \bar{X}$, which is closed under conjugation and such that the restriction map yields a monomorphism $\operatorname{Pic}(\bar{X}) \hookrightarrow$ $\prod_{\bar{C} \in \bar{S}} \operatorname{Pic}(\bar{C})$. Then $\alpha_{\emptyset}$ is the class of the extension (3.12):

$$
0 \longrightarrow \operatorname{Pic}(\bar{X}) \longrightarrow \prod_{\bar{C} \in \bar{S}} \operatorname{Pic}(\bar{C}) \longrightarrow \tilde{\operatorname{Br}}_{\bar{S}}(\bar{X}) \longrightarrow \operatorname{Br}(\bar{X}) \longrightarrow 0 .
$$

Without loss of generality, we may assume that the curves of the set $\bar{S}$ generate the group $\operatorname{Num}_{1}(\bar{X})$ and that $\bar{S}$ contains at least one smooth iterated hyperplane section of $\bar{X}$. We show that the map $\operatorname{Pic}(\bar{X}) \rightarrow \prod_{\bar{C} \in \bar{S}} \operatorname{Pic}(\bar{C})$ has a left inverse in the $\mathbb{Q}$ localized category of the category of discrete $G_{k}$-modules, and this settles the proof. 
We have a commutative, exact, self-defining diagram of discrete $G_{k}$-modules

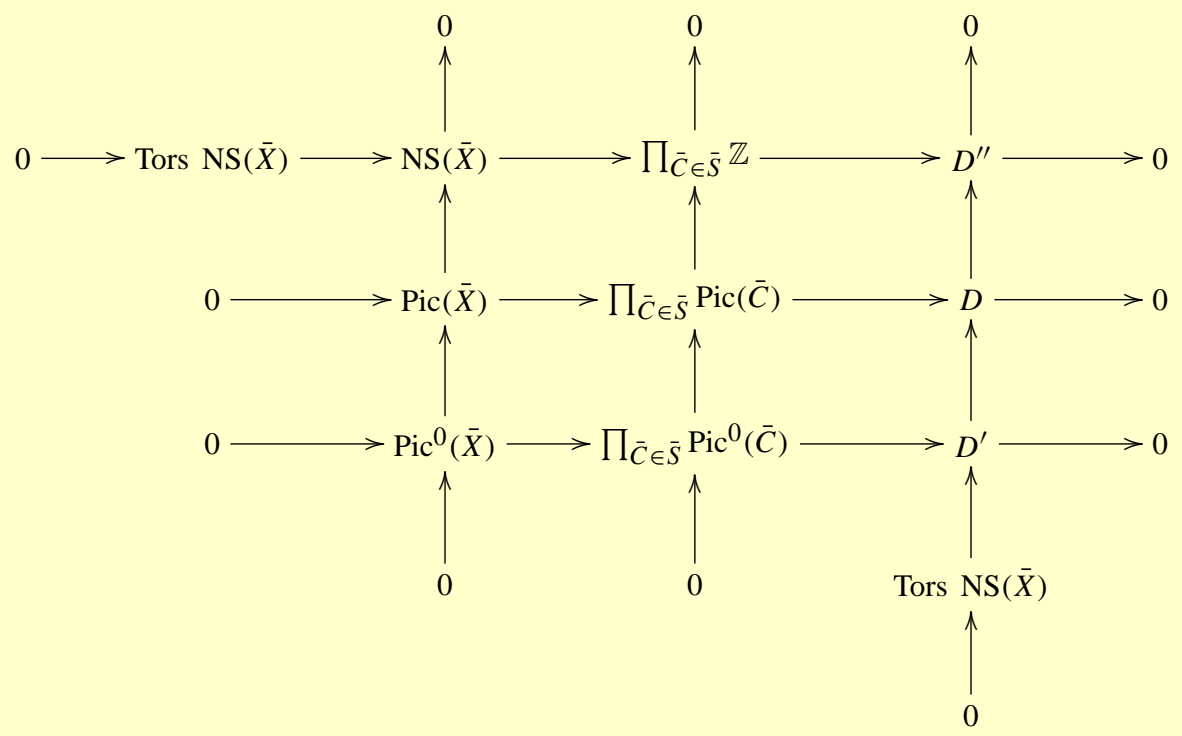

Since the group Tors $\mathrm{NS}(\bar{X})$ has in particular a finite exponent, this diagram yields a 9-lemma diagram in the $\mathbb{Q}$-localized category, in which, by Remark 3.9, the two middle columns (in particular) and the top row are split. It suffices then to show that the same thing holds for the bottom row to conclude the desired result. Let $\bar{C} \in \bar{S}$ be a smooth iterated hyperplane section of $\bar{X}$. One has an isogeny $\operatorname{Pic}^{0}(\bar{X}) \rightarrow$ $\operatorname{Pic}^{0}(\bar{C}) \stackrel{\sim}{\longrightarrow} J(\bar{C}) \rightarrow \operatorname{Alb}(\bar{X})$, and by composition with the inverse isogeny, we deduce an abstract group-theoretical $\mathbb{Q}$-left inverse for $\operatorname{Pic}^{0}(\bar{X}) \rightarrow \operatorname{Pic}^{0}(\bar{C})$. Summing then over the finite set of conjugates of this map, one easily constructs the claimed inverse.

We end this section by proving the following (see Remark 3.5(a); see also Remark 5.5).

PROPOSITION 3.17

In the notation at the beginning of Section 3 , if $[z]=0$, then $\Delta_{n}^{2}(z)=0$.

\section{Proof}

Since $\Delta_{n}^{2}$ is additive (see Remark 3.5(d)), we may assume that $z=\operatorname{div}_{C}(f)$ with $C \subset$ $X$ a reduced irreducible curve and $f \in k(C)^{*}$. To shortcut discussions, we reduce the question to the particular case where $C$ is a geometrically integral curve. To this end, observe that, if $\bar{C}_{1}, \ldots, \bar{C}_{r}$ are the reduced curves supported by the irreducible components of the subscheme $\bar{C} \subset \bar{X}$, then there exist $\bar{f}_{i} \in \bar{k}\left(\bar{C}_{i}\right)^{*}, i=1, \ldots, r$, 
such that $\bar{z}=\sum_{i=1}^{r} \operatorname{div}_{\bar{C}_{i}}\left(\bar{f}_{i}\right)$. Let $k \subset k_{1} \subset \bar{k}$ be a finite extension such that all of the $\bar{C}_{i}$ and the $\bar{f}_{i}$ are defined over $k_{1}$. Writing for a moment $X_{1}=X \otimes_{k} k_{1}$ and writing $F^{2} Z_{0}(X)$ for the inverse image of $T(X) \subset \mathrm{CH}_{0}(X)$ in $Z_{0}(X)$, we have a commutative diagram

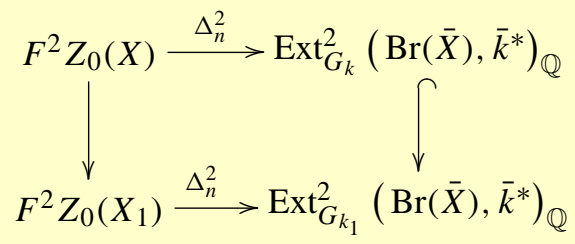

where we have included the statement that the right-hand-side vertical map is injective. (This accounts for the claimed reduction to the case of a geometrically integral curve $C$.) Indeed, if $G$ is a profinite group, $H$ is a closed subgroup of finite index $d$, and $M$ and $N$ are discrete $G$-modules, then, for all $i \geq 0$, the kernel of the map $\operatorname{Ext}_{G}^{i}(M, N) \rightarrow \operatorname{Ext}_{H}^{i}(M, N)$ is killed by $d$. Namely, in the notation of [S, pages I-12 and I-13], if [ $\left.E^{\cdot}\right] \in \operatorname{Ext}_{G}^{i}(M, N)$, then one has natural morphisms of exact sequences of discrete $G$-modules $E^{\cdot} \rightarrow M_{G}^{H}\left(E^{\cdot}\right)$ and $M_{G}^{H}\left(E^{\cdot}\right) \rightarrow E^{\cdot}$ such that the composition $E^{\cdot} \rightarrow E^{\cdot}$ is the multiplication by $d$. If $\left[E^{\cdot}\right]=0$ in $\operatorname{Ext}_{H}^{i}(M, N)$, then $\left[M_{G}^{H}\left(E^{\cdot}\right)\right]=0$ in $\operatorname{Ext}_{G}^{i}(M, N)$ and whence $d\left[E^{*}\right]=0$ in $\operatorname{Ext}_{G}^{i}(M, N)$.

Thus suppose now that the curve $C$ is geometrically integral. Let $N \stackrel{\nu}{\rightarrow} C$ be the normalization of $C$. This is a geometrically irreducible smooth projective curve over $k$. We have $z=v_{*}\left(z_{N}\right)$ with $z_{N}=\operatorname{div}_{N}(f)$. Write $Z_{N}=\left|z_{N}\right|$ for the support of $z_{N}$, and write $\bar{Z}_{N}$ for the inverse image of this set in $\bar{N}$. One has a commutative diagram

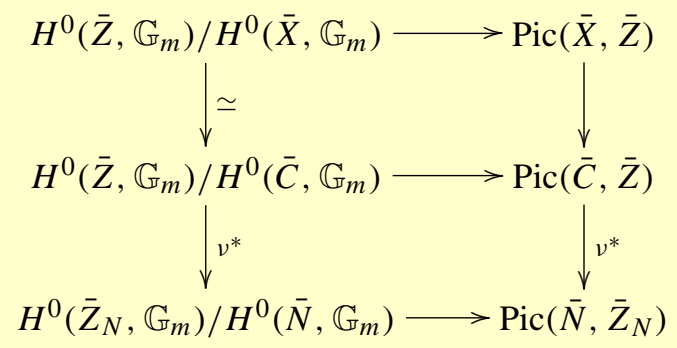

the upper vertical maps being the restriction maps. We recall from [CTS, page 424] the way to construct in this case a natural $G_{k}$-morphism $\widetilde{z}_{N}: \operatorname{Pic}\left(\bar{N}, \bar{Z}_{N}\right) \rightarrow \bar{k}^{*}$ (see (3.1)) extending the morphism $z_{N}: H^{0}\left(\bar{Z}_{N}, \mathbb{G}_{m}\right) / H^{0}\left(\bar{N}, \mathbb{G}_{m}\right) \rightarrow \bar{k}^{*}$ (see (2.3)). Given $[(\bar{L}, \bar{\theta})] \in \operatorname{Pic}\left(\bar{N}, \bar{Z}_{N}\right)$, let $\bar{Z}_{N} \subset \bar{U} \subset \bar{N}$ be an open subset such that $\bar{L}$ is trivial over $\bar{U}$. Choose an isomorphism $\bar{\Phi}: \mathscr{O}_{\bar{U}} \stackrel{\sim}{\longrightarrow} \bar{L} \otimes \mathscr{O}_{\bar{U}}$, and let $\bar{\sigma} \in H^{0}(\bar{N}, \bar{L} \otimes$ $\mathscr{K}_{\bar{N}}$ ) be the rational section of $\bar{L}$ that it defines. The trivialization $\bar{\theta}: \mathscr{O}_{\bar{Z}_{N}} \stackrel{\sim}{\longrightarrow}$ 
$\bar{L} \otimes \mathscr{O}_{\bar{Z}_{N}}$ allows us to view $\bar{\sigma}$ as a true function at the points of $\bar{Z}_{N}$. One puts then

$$
\widetilde{z}_{N}[(\bar{L}, \bar{\theta})]=\frac{\bar{f}(\operatorname{div}(\bar{\sigma}))}{\bar{\sigma}(\operatorname{div}(\bar{f}))}
$$

(of course, $\operatorname{div}(\bar{f})=\bar{z}_{N}$ ). The result is independent of the choice of $\bar{\Phi}$ due to Weil reciprocity (see [CTS, page 424]), and $\widetilde{z}_{N}$ restricts indeed to $z_{N}$ on $H^{0}\left(\bar{Z}_{N}, \mathbb{G}_{m}\right) / H^{0}\left(\bar{N}, \mathbb{G}_{m}\right)$.

We put $\widetilde{z}_{C}=\widetilde{z}_{N} \circ v^{*}$ and take now $\widetilde{z}$ in (3.1) as the composition of the restriction $\operatorname{map} \operatorname{Pic}(\bar{X}, \bar{Z}) \rightarrow \operatorname{Pic}(\bar{C}, \bar{Z})$ with $\widetilde{z}_{C}$. Let $\bar{S}$ be a finite set of closed irreducible curves of $\bar{X}$ adequate for $Z$ and containing the curve $\bar{C}$. The commutative diagram

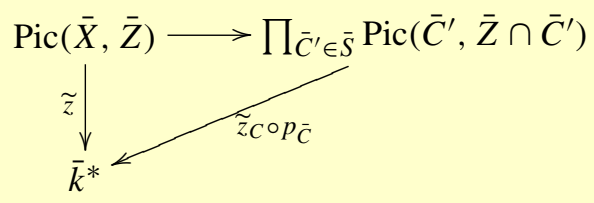

with $p_{\bar{C}}$ the projection map $\prod_{\bar{C}^{\prime} \in \bar{S}} \operatorname{Pic}\left(\bar{C}^{\prime}, \bar{Z} \cap \bar{C}^{\prime}\right) \rightarrow \operatorname{Pic}(\bar{C}, \bar{Z})$ now implies (see (3.14) and (3.15)) that $\widetilde{z}\left(\alpha_{Z}\right)=0$.

\section{A cohomological description of $\alpha_{Z}$}

We keep the assumptions and notation from Section 3. The exact sequence of $G_{k^{-}}$ modules (3.10) hints at the exact sequence of sheaves on $\bar{X}$,

$$
0 \longrightarrow \mathbb{G}_{m, \bar{X}} \longrightarrow \prod_{\bar{C} \subset \bar{X}} \mathbb{G}_{m, \bar{C}} \longrightarrow \overline{\mathscr{G}} \longrightarrow 0
$$

where the product in the second term is taken over all closed irreducible curves $\bar{C} \subset \bar{X}$ and where $\overline{\mathscr{G}}$ stands for the quotient sheaf. If cohomology were to commute with infinite products (which it does not), then, by Proposition (3.11), the sequence (3.10) would follow from the cohomology sequence of (4.1), modulo comparison of $\widetilde{\operatorname{Br}}(\bar{X})$ with $H^{1}(\bar{X}, \overline{\mathscr{G}})$. We get this, nevertheless, by working with finite sets $\bar{S}$ of (closed, irreducible) curves on $\bar{X}$. However, in this case the map $\mathbb{G}_{m, \bar{X}} \rightarrow \prod_{\bar{C} \in \bar{S}} \mathbb{G}_{m, \bar{C}}$ is not injective, and we must interpret things accordingly.

Let $Z \subset X$ be a 0 -dimensional closed subset. Let $\bar{S}$ be a finite set of closed irreducible curves of $\bar{X}$ adequate for $Z$ (see Section 3). The restriction map

$$
\mathbb{G}_{m, \bar{X}, \bar{Z}} \longrightarrow \prod_{\bar{C} \in \bar{S}} \mathbb{G}_{m, \bar{C}, \bar{Z} \cap \bar{C}}
$$

yields a two-term complex, and we consider the long exact sequence deduced from the first hypercohomology spectral sequence of this complex. This is the same as the 
long exact sequence of hypercohomology of the exact sequence of complexes

$$
0 \longrightarrow\left(\prod_{\bar{C} \in \bar{S}} \mathbb{G}_{m, \bar{C}, \bar{Z} \cap \bar{C}}\right)[-1] \longrightarrow(4.2) \longrightarrow \mathbb{G}_{m, \bar{X}, \bar{Z}}[0] \longrightarrow 0 .
$$

PROPOSITION 4.4

Let $\bar{S}$ be chosen as above. Then the exact sequence (3.14) is obtained by restriction to $\operatorname{Br}(\bar{X})$, from the long exact sequence of hypercohomology of (4.3).

\section{Proof}

From the exact sequences of type (2.2) which define these sheaves, we deduce the equalities (see also (2.1) and (3.11)) $H^{1}\left(\bar{X}, \mathbb{G}_{m, \bar{X}}, \bar{Z}\right)=\operatorname{Pic}(\bar{X}, \bar{Z})$, $H^{2}\left(\bar{X}, \mathbb{G}_{m, \bar{X}, \bar{Z}}\right)=H^{2}\left(\bar{X}, \mathbb{G}_{m}\right), H^{1}\left(\bar{X}, \prod_{\bar{C} \in \bar{S}} \mathbb{G}_{m, \bar{C}, \bar{Z} \cap \bar{C}}\right)=\prod_{\bar{C} \in \bar{S}} \operatorname{Pic}(\bar{C}, \bar{Z} \cap \bar{C})$, and $H^{2}\left(\bar{X}, \prod_{\bar{C} \in \bar{S}} \mathbb{G}_{m, \bar{C}, \bar{Z} \cap \bar{C}}\right)=0$. We extract, therefore, from the hypercohomology sequence of (4.3) an exact sequence

$$
\operatorname{Pic}(\bar{X}, \bar{Z}) \longrightarrow \prod_{\bar{C} \in \bar{S}} \operatorname{Pic}(\bar{C}, \bar{Z} \cap \bar{C}) \longrightarrow \mathbb{H}^{2}(4.2) \longrightarrow H^{2}\left(\bar{X}, \mathbb{G}_{m}\right) \longrightarrow 0 .
$$

The first map in (4.5) is the restriction map, whence, by the very hypothesis on $\bar{S}$, it is injective. The rest of the statement follows then by exhibiting a morphism of $G_{k}$-modules $\widetilde{\operatorname{Br}}_{\bar{S}}(\bar{X}, \bar{Z}) \rightarrow \mathbb{H}^{2}(4.2)$, compatible with the identity map of $\prod_{\bar{C} \in \bar{S}} \operatorname{Pic}(\bar{C}, \bar{Z} \cap \bar{C})$ and the embedding map $\operatorname{Br}(\bar{X}) \hookrightarrow H^{2}\left(\bar{X}, \mathbb{G}_{m}\right)$. We give an explicit recipe, leaving the details to the reader. The exact diagram

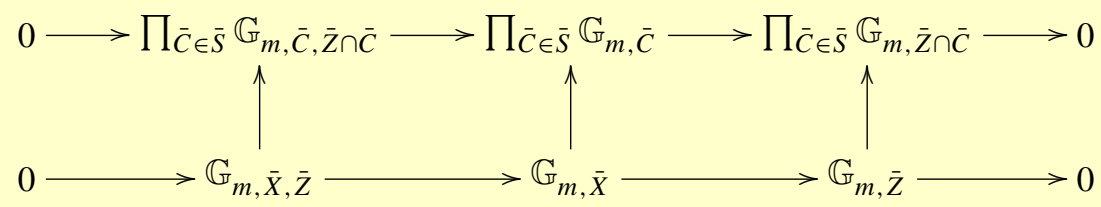

yields a quasi-isomorphism between the complex (4.2) and the total complex associated with the double complex deduced from the right-hand-side square:

$$
0 \longrightarrow \mathbb{G}_{m, \bar{X}} \stackrel{\left(\begin{array}{l}
1 \\
1
\end{array}\right)}{\longrightarrow}\left(\prod_{\bar{C} \in \bar{S}} \mathbb{G}_{m, \bar{C}}\right) \oplus \mathbb{G}_{m, \bar{Z}} \stackrel{(1,-1)}{\longrightarrow} \prod_{\bar{C} \in \bar{S}} \mathbb{G}_{m, \bar{Z} \cap \bar{C}} \longrightarrow 0 .
$$

Here we have indicated schematically the way in which the arrows are constructed from the corresponding ones in the previous diagram.

Now let $(\bar{P}, \bar{\xi}, \bar{f}, \bar{\varphi})$ be a 4-tuple representing an element of $\widetilde{\operatorname{Br}}_{\bar{S}}(\bar{X}, \bar{Z})$. We attach to this object a 2-hypercocycle for a suitable open covering of $\bar{X}$ (in the étale topology), with values in the above complex. This defines then the map sought. Let $\overline{\mathscr{U}}=\left(\bar{U}_{i}\right)_{i \in I}$ be an open covering of $\bar{X}$ trivializing the Severi-Brauer variety $\bar{P}$. Call 
$n-1$ the relative dimension of $\bar{P}$ over $\bar{X}$. For all $i \in I$, choose an isomorphism $\bar{h}_{i}: \mathbb{P}_{\bar{U}_{i}}^{n-1} \stackrel{\sim}{\longrightarrow} \bar{P}_{\bar{U}_{i}}$. For all $i, j \in I$, let

$$
\bar{\theta}_{i j}: \mathscr{O}_{\mathbb{P}_{U_{i j}}^{n-1}}(1) \stackrel{\sim}{\longrightarrow} \mathscr{O}_{\mathbb{P}_{U_{i j}}^{n-1}}(1)
$$

be a relative isomorphism over the isomorphism $\bar{h}_{i}^{-1} \bar{h}_{j}: \mathbb{P}_{\bar{U}_{i j}}^{n-1} \stackrel{\sim}{\longrightarrow} \mathbb{P}_{\bar{U}_{i j}}^{n-1}$. The formula $\bar{\alpha}_{i j k}=\left(\bar{\theta}_{j k} \bar{\theta}_{i k}^{-1} \bar{\theta}_{i j}\right)^{-1}$ defines then a 2-cocycle $\bar{\alpha} \in Z^{2}\left(\overline{\mathscr{U}}, \mathbb{G}_{m, \bar{X}}\right)$ representing $[\bar{P}] \in \operatorname{Br}(\bar{X})$ (see [Mi1, pages 123 and 143]; see also Remark 4.11(b)). Given $\bar{C} \in \bar{S}$ and $i \in I$, the inverse image of $\bar{\xi}_{\bar{C}} \otimes \mathscr{O}_{\bar{C} \cap \bar{U}_{i}}$ by $\bar{h}_{i}$ is isomorphic with $\mathscr{O}_{\mathbb{P}_{\bar{C} \cap \bar{U}_{i}}^{n-1}}$ (1) times an invertible sheaf coming from $\bar{C} \cap \bar{U}_{i}$. Upon refining the covering $\overline{\mathscr{U}}$ suitably, we may ensure that the latter invertible sheaf is trivial for all $\bar{C} \in \bar{S}$ and all $i \in I$. Then, for all $\bar{C} \in \bar{S}$ and all $i \in I$, let

$$
\bar{\mu}_{i, \bar{C}}: \mathscr{O}_{\mathbb{P}_{\bar{C} \cap \bar{U}_{i}}^{n-1}}(1) \stackrel{\sim}{\longrightarrow} \bar{\xi}_{\bar{C}} \otimes \mathscr{O}_{\bar{C} \cap \bar{U}_{i}}
$$

be a relative isomorphism over the restriction of $\bar{h}_{i}$. The formula $\bar{\mu}_{i, \bar{C}}^{-1} \bar{\mu}_{j, \bar{C}}=\bar{\beta}_{i j, \bar{C}} \bar{\theta}_{i j}$ defines then a 1-cochain $\bar{\beta}=\left\{\bar{\beta}_{i j, \bar{C}}\right\} \in C^{1}\left(\overline{\mathscr{U}}, \prod_{\bar{C} \in \bar{S}} \mathbb{G}_{m, \bar{C}}\right)$, and $d \bar{\beta}$ is the image of $\bar{\alpha}$. Next comes $\bar{f}$. For all $i \in I$, let

$$
\bar{\theta}_{i}: \mathscr{O}_{\mathbb{P}_{\bar{Z} \cap \bar{U}_{i}}^{n-1}}(1) \stackrel{\sim}{\longrightarrow} \mathscr{O}_{\mathbb{P}_{\bar{Z}}^{n-1} \bar{U}_{i}}
$$

be a relative isomorphism over the isomorphism $\bar{f}^{-1} \circ \bar{h}_{i}: \mathbb{P}_{\bar{Z} \cap \bar{U}_{i}}^{n-1} \stackrel{\sim}{\longrightarrow} \mathbb{P}_{\bar{Z} \cap \bar{U}_{i}}^{n-1}$. We define a 1-cochain $\bar{\gamma}=\left\{\bar{\gamma}_{i j}\right\} \in C^{1}\left(\overline{\mathscr{U}}, \mathbb{G}_{m, \bar{Z}}\right)$ by the formula $\bar{\theta}_{i}^{-1} \bar{\theta}_{j}=\bar{\gamma}_{i j} \bar{\theta}_{i j}$, and $d \bar{\gamma}$ is the image of $\bar{\alpha}$. Finally, we consider $\bar{\varphi}$. For all $\bar{C} \in \bar{S}$ and all $i \in I$, the formula $\bar{\varphi}_{\bar{C}}^{-1} \bar{\mu}_{i, \bar{C}}=\bar{\varepsilon}_{i, \bar{C}} \bar{\theta}_{i}$ above $\bar{Z} \cap \bar{C} \cap \bar{U}_{i}$ defines a 0-cochain $\bar{\varepsilon}=\left\{\bar{\varepsilon}_{i, \bar{C}}\right\} \in$ $C^{0}\left(\overline{\mathscr{U}}, \prod_{\bar{C} \in \bar{S}} \mathbb{G}_{m, \bar{Z} \cap \bar{C}}\right)$. The coboundary $d \bar{\varepsilon}$ equals the image of $\bar{\beta}$ minus the image of $\bar{\gamma}$. Taken together, $(\bar{\alpha}, \bar{\beta} \oplus \bar{\gamma}, \bar{\varepsilon})$ yield then the 2-hypercocycle defining the image of $[(\bar{P}, \bar{\xi}, \bar{f}, \bar{\varphi})]$ in $\mathbb{H}^{2}(4.2)$.

By completing the exact sequence (4.5) with zero on the left, we obtain a 2extension representing an element in $\operatorname{Ext}_{G_{k}}^{2}\left(H^{2}\left(\bar{X}, \mathbb{G}_{m}\right), \operatorname{Pic}(\bar{X}, \bar{Z})\right)$ which depends only on $Z$ and which we denote again by $\alpha_{Z}$. Then we may repeat the procedure from Section 3, starting with Definition 3.4 applied to this setting. This yields the map $\Delta_{n}^{2}$ from (1.7), modulo the analogue of Remark 3.5. Furthermore, the proof of (3.16) applies here too, showing that $\alpha_{\emptyset}=0$ in $\operatorname{Ext}_{G_{k}}^{2}\left(H^{2}\left(\bar{X}, \mathbb{G}_{m}\right), \operatorname{Pic}(\bar{X})\right)_{\mathbb{Q}}$. And, finally, Proposition 3.17 carries over without change, thereby completing the construction of $\Delta_{n}^{2}$ from (1.7).

In Section 5 we compare this map $\Delta_{n}^{2}$ with the higher Abel-Jacobi map $d_{n}^{2}$ in $l$ adic theory. This happens through the computation of the maps $d_{j}^{2}$ made by $\mathrm{U}$. Jannsen 
in [J4]. A main role in that computation is played by the 2-extensions $\chi_{i}\left(C^{\cdot}\right)$,

$$
0 \longrightarrow Z^{i} / B^{i} \longrightarrow C^{i} / B^{i} \longrightarrow Z^{i+1} \longrightarrow Z^{i+1} / B^{i+1} \longrightarrow 0,
$$

attached to a complex $\left(C^{*}\right)$ in an abelian category $\mathscr{C}$, and whose equivalence class in $\operatorname{Ext}_{\mathscr{C}}^{2}\left(H^{i+1}\left(C^{\cdot}\right), H^{i}\left(C^{*}\right)\right)$ depends only on the image of $\left(C^{\cdot}\right)$ in the derived category (see [J4, page 263]).

We denote by $h: X \rightarrow \operatorname{Spec}(k)$ the structure map. We introduce also a ring $R$ to be $R=\mathbb{Z}$ if $k$ is a perfect field and $R=\mathbb{Z}[1 / p], p=\operatorname{char}(k)$, otherwise. Let $Z \subset X$ be a 0 -dimensional closed subset. Let $S$ be a finite set of closed irreducible curves of $X$ such that the set $\bar{S}$ of the irreducible components of their inverse images in $\bar{X}$ is adequate for $Z$. We consider the two-term complex of sheaves on $X$ analogous to (4.2),

$$
\mathbb{G}_{m, X, Z} \longrightarrow \prod_{C \in S} \mathbb{G}_{m, C, Z \cap C},
$$

and the exact sequence of complexes of sheaves on $X$, similar to (4.3), built from it,

$$
0 \longrightarrow\left(\prod_{C \in S} \mathbb{G}_{m, C, Z \cap C}\right)[-1] \longrightarrow(4.6) \longrightarrow \mathbb{G}_{m, X, Z}[0] \longrightarrow 0 .
$$

Upon taking base change from $k$ to $\bar{k}$ in (4.6) and (4.7), the result maps naturally to (4.2) and (4.3), respectively. As a consequence, we have a commutative exact diagram of discrete $G_{k}$-modules

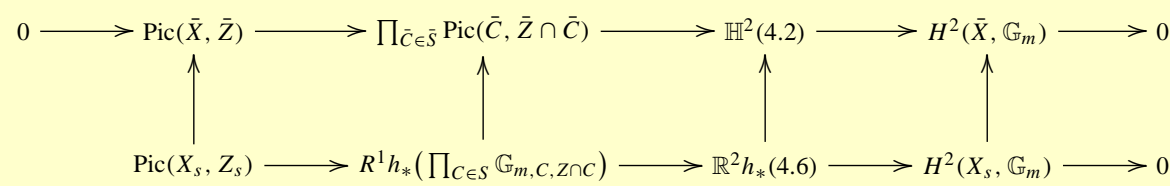

where the bottom row comes from the higher direct images sequence of (4.7) for $h ; k \subset k_{s} \subset \bar{k}$ is the separable closure of $k ; X_{s}=X \otimes_{k} k_{s} ; Z_{s} \subset X_{s}$ is the 0dimensional closed subset obtained as the inverse image of $Z$ in $X_{s}$; and $S_{s}$ is the set of closed irreducible curves of $X_{S}$ obtained from the inverse images of the curves $C \in S$. The zero term at the right end of the bottom sequence is due to the vanishing of $R^{2} h_{*}\left(\prod_{C \in S} \mathbb{G}_{m, C, Z \cap C}\right)$, which in turn follows from Proposition 3.11 together with the fact that the quotient sheaf of $\left(\prod_{C \in S} \mathbb{G}_{m, C, Z \cap C}\right) \otimes_{k} k_{s} \hookrightarrow \prod_{C_{s} \in S_{s}} \mathbb{G}_{m, C_{s}, Z_{s} \cap C_{s}}$ on $X_{s}$ has finite support. From (4.8) we deduce the exact diagram (the subscript $R$ 
standing for $\otimes_{\mathbb{Z}} R$ )
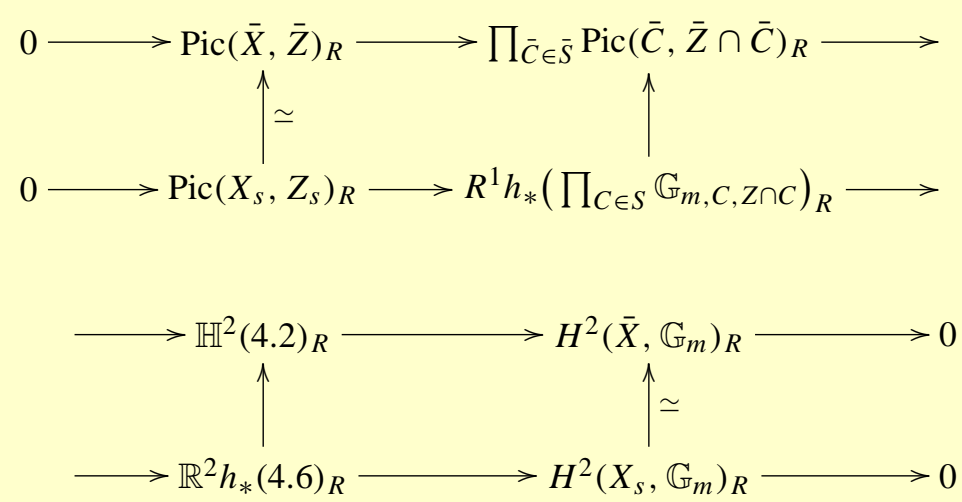

The vertical isomorphism on the left-hand side follows from the isomorphism $\operatorname{Pic}\left(X_{S}\right)_{R} \stackrel{\sim}{\longrightarrow} \operatorname{Pic}(\bar{X})_{R}$, and the right-hand-side vertical isomorphism follows from this and by proper base change.

PROPOSITION 4.10

Let, as before, $Z \subset X$ be a 0 -dimensional closed subset, and let $S$ be a finite set of closed irreducible curves of $X$ such that $\bar{S}$ is adequate for $Z$. Then one has, in $\operatorname{Ext}_{G_{k}}^{2}\left(H^{2}\left(\bar{X}, \mathbb{G}_{m}\right)_{R}, \operatorname{Pic}(\bar{X}, \bar{Z})_{R}\right),\left(\alpha_{Z}\right)_{R}=-\chi_{1}\left(\mathrm{Rh}_{*} \mathbb{G}_{m, X, Z}\right)_{R}$.

\section{Proof}

Let

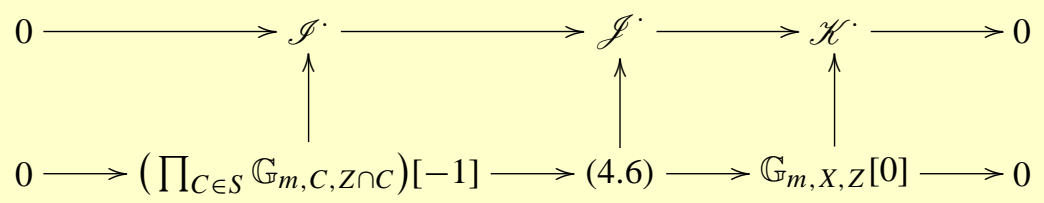

be a morphism of exact sequences of complexes of sheaves on $X$, with the sheaves in the upper row injective and the vertical maps being quasi-isomorphisms. To simplify the notation, we write here $I^{i}=\left(h_{*} \mathscr{I}^{i}\right)_{R}$, and similarly for $\mathscr{J} \cdot$ and $\mathscr{K}$. We have an 
exact commutative diagram

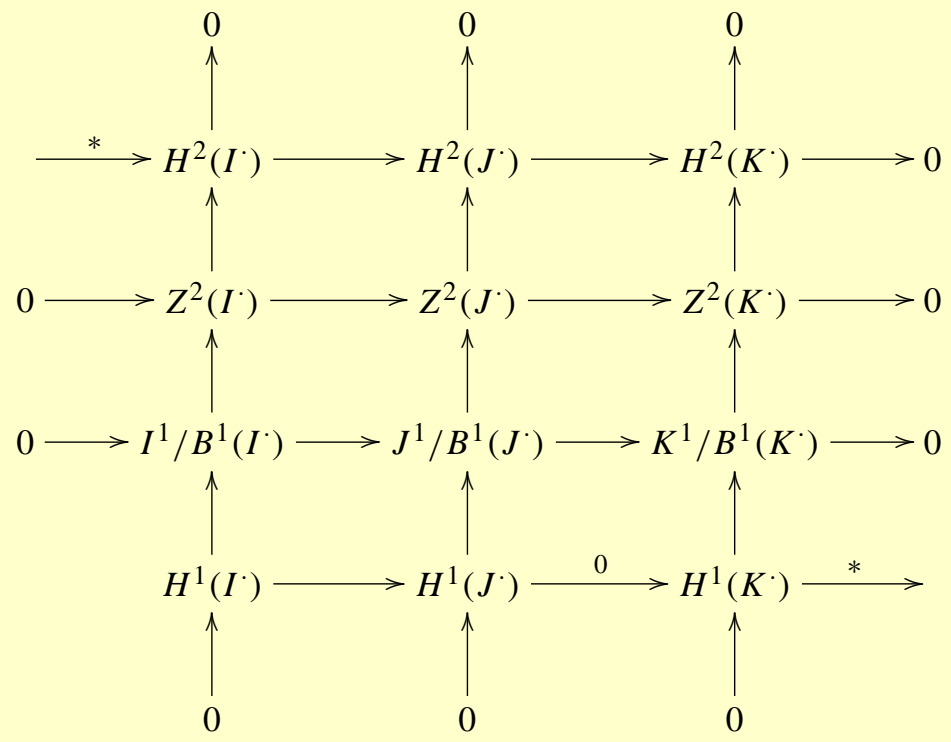

the arrow marked with a star being the map given by the snake lemma. The exact sequence

$$
0 \longrightarrow H^{1}\left(K^{*}\right) \stackrel{*}{\longrightarrow} H^{2}\left(I^{*}\right) \longrightarrow H^{2}\left(J^{*}\right) \longrightarrow H^{2}\left(K^{*}\right) \longrightarrow 0
$$

is the bottom row of diagram (4.9), whence, by that diagram and Proposition 4.4, it represents $\left(\alpha_{Z}\right)_{R}$. Furthermore, the right-hand-side column in the above diagram represents $\chi_{1}\left(\mathrm{Rh}_{*} \mathbb{G}_{m, X, Z}\right)_{R}$. If we replace, in that diagram, $I^{1} / B^{1}\left(I^{\cdot}\right)$ and $J^{1} / B^{1}\left(J^{\cdot}\right)$ by their images in $Z^{2}\left(I^{\cdot}\right)$ and $Z^{2}\left(J^{\cdot}\right)$, respectively, and both $H^{1}\left(I^{\cdot}\right)$ and $H^{1}\left(J^{\cdot}\right)$ by zero, then we obtain a new exact diagram in which the two exact sequences consid- 
ered remain unchanged, and which looks as follows:

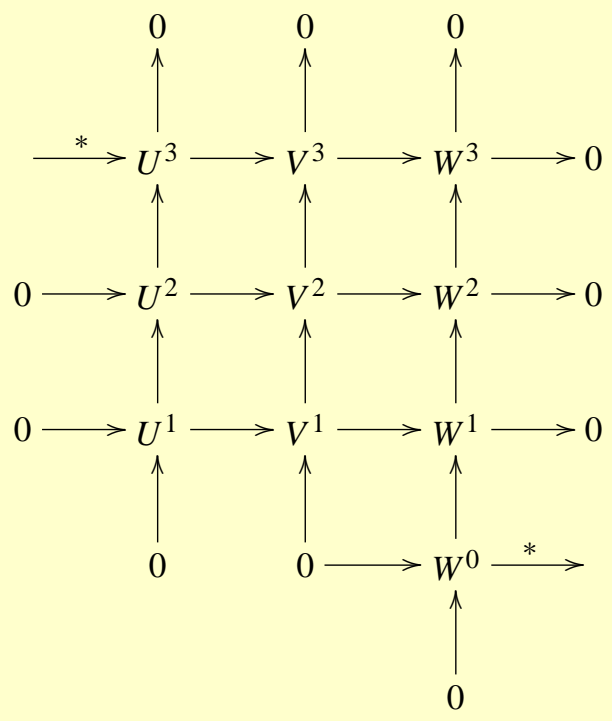

Writing this equivalently as

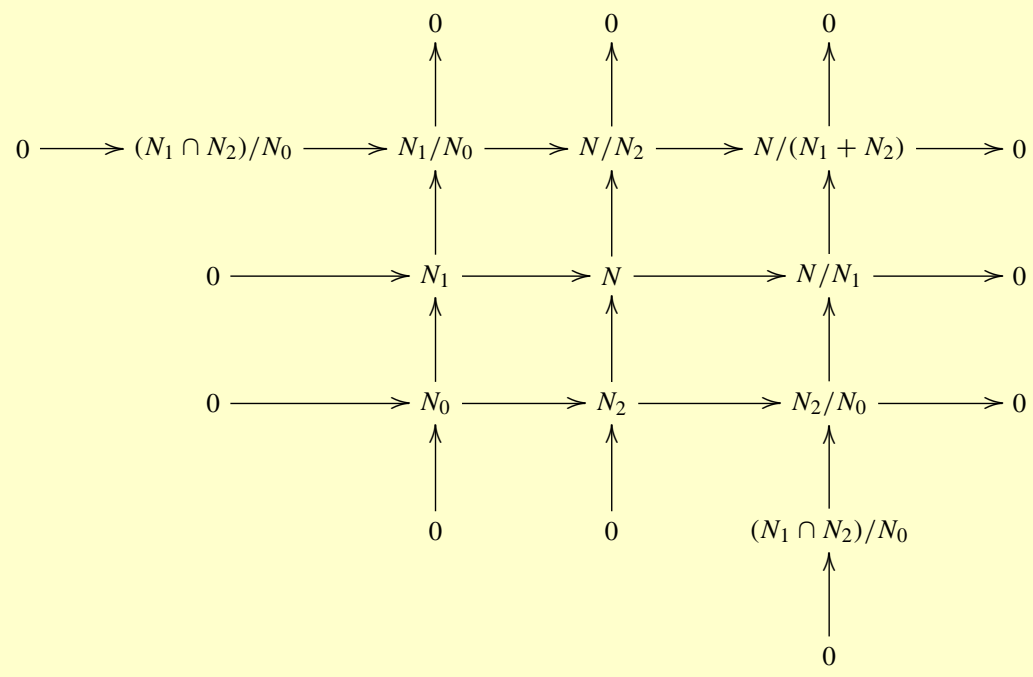

we find that the two longer exact sequences indeed yield opposite extension classes since they are obtained by composing the outer short exact sequences, in the two 
possible ways, in the following 9-lemma diagram:

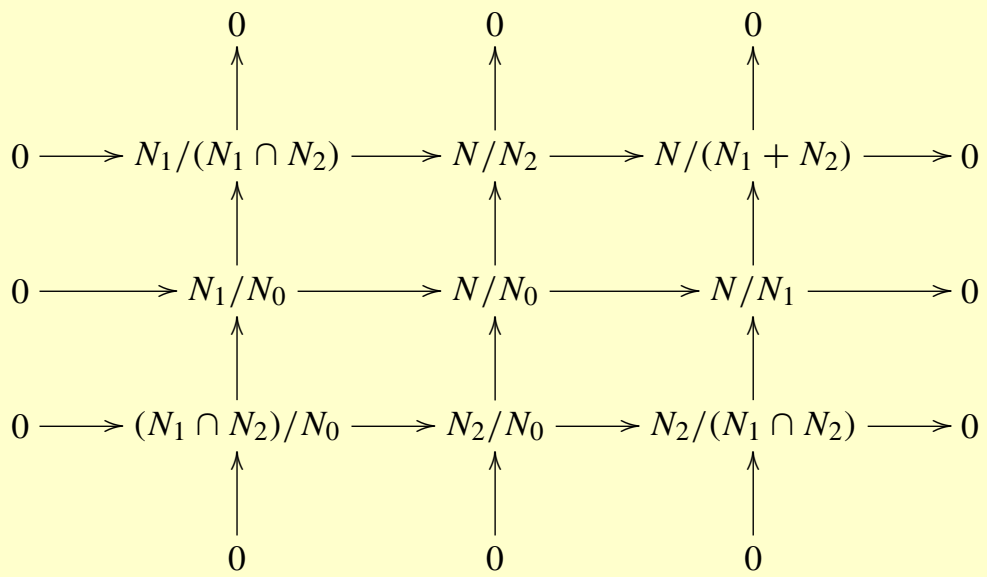

Remark 4.11

(a) There might be an elementary general principle of abstract nonsense at work in Proposition 4.10. We have not looked further into this, except for the (present) remark that the same proof applies to compute $\chi_{0}\left(\mathrm{Rh}_{*} \mathbb{G}_{m, Y}\right)$, where $Y$ denotes again a smooth, projective, geometrically irreducible variety over a field $k$. Namely, if $T \subset Y$ is any nonempty finite closed subset, one finds that $-\chi_{0}\left(\mathrm{Rh}_{*} \mathbb{G}_{m, Y}\right) \in$ $\operatorname{Ext}_{G_{k}}^{2}\left(\operatorname{Pic}\left(Y_{s}\right), k_{s}^{*}\right)$ is represented by the exact sequence

$$
0 \longrightarrow k_{s}^{*} \longrightarrow H^{0}\left(T_{s}, \mathbb{G}_{m}\right) \longrightarrow \operatorname{Pic}\left(Y_{s}, T_{s}\right) \longrightarrow \operatorname{Pic}\left(Y_{s}\right) \longrightarrow 0
$$

(see also Section 2). The extension (4.12) is not zero in general because taking cup product with $\chi_{0}\left(\mathrm{Rh}_{*} \mathbb{G}_{m, Y}\right)$ yields differentials of the Lyndon-Hochschild-Serre spectral sequence $H^{i}\left(G_{k}, H^{j}\left(Y_{s}, \mathbb{G}_{m}\right)\right) \Rightarrow H^{i+j}\left(Y, \mathbb{G}_{m}\right)$ (see [J4, page 264]) and in particular, one has the exact sequence

$$
0 \longrightarrow \operatorname{Pic}(Y) \longrightarrow \operatorname{Pic}\left(Y_{s}\right)^{G_{k}} \stackrel{\cup \chi_{0}}{\longrightarrow} \operatorname{Br}(k) \longrightarrow \operatorname{Br}(Y) .
$$

On the other hand, $\chi_{0}\left(\mathrm{Rh}_{*} \mathbb{G}_{m, Y}\right)$ is a torsion element killed by the greatest common divisor of the integers $d$ such that $Y$ contains a closed point $y$ of degree $d$ over the base field $k$. Note indeed that, in the preceding notation, taking $T=\{y\}$, we have a morphism of $G_{k}$-modules $H^{0}\left(T_{s}, \mathbb{G}_{m}\right) \rightarrow k_{s}^{*}$ inducing multiplication by $d$ in $k_{s}^{*}$.

(b) If $Y$ is a Severi-Brauer variety over $k$, then $\operatorname{Pic}\left(Y_{S}\right)^{G_{k}}=\operatorname{Pic}\left(Y_{S}\right)=\mathbb{Z}$, and the image of $1 \in \mathbb{Z}$ in (4.13) is commonly claimed in the literature (without proof) to equal the class $[Y] \in \operatorname{Br}(k)$. This is in contrast with [Gr3, p. 129, lines 4-12], 
where it is claimed (also without proof) to be equal to $\left[Y^{\vee}\right]=-[Y]$. The first of these claims is equivalent to the statement that the sequence (4.12) represents in this case $-[Y] \in \operatorname{Br}(k)$. By direct computation, we show that this is indeed so.

As in the proof of Theorem 7.8 (see Section 7), let $\xi_{s}$ be an invertible sheaf of degree 1 on $Y_{s}$, and choose, for all $\sigma \in G_{k}$, a relative isomorphism $\widetilde{\sigma}: \xi_{s} \stackrel{\sim}{\longrightarrow} \xi_{s}$ over the isomorphism $\sigma: Y_{s} \stackrel{\sim}{\longrightarrow} Y_{s}$. Then the class of the extension (4.12) in $\operatorname{Br}(k)$ is represented by the 2-cocycle $\rho^{\prime}$ defined by the formula $\rho^{\prime}(\sigma, \tau)=\widetilde{\sigma}^{-1} \widetilde{\tau}^{-1}(\widetilde{\sigma \tau})$ for all $\sigma, \tau \in G_{k}$. On the other hand, if $n-1=\operatorname{dim} Y_{s}$ and $f: \mathbb{P}_{k_{s}}^{n-1} \stackrel{\sim}{\longrightarrow} Y_{s}$ is any isomorphism over $k_{s}$, then the class of $Y_{s}$ in $H^{1}\left(G_{k}\right.$, Aut $\left._{k_{s}}\left(\mathbb{P}_{k_{s}}^{n-1}\right)\right)$ is represented by the 1-cocycle defined by $\psi(\sigma)=f^{-1} \sigma^{-1} f \sigma_{0}$ for all $\sigma \in G_{k}$. (In the right-hand-side member of this formula, $\sigma$ represents the automorphism of $Y_{s}$ defined by $\sigma$, while $\sigma_{0}$ is the automorphism that $\sigma$ defines in $\mathbb{P}_{k_{s}}^{n-1}$.) Then, the class of $Y_{s}$ in $\operatorname{Br}(k)$ is minus the image of $[\psi]$ by the connecting map for the exact sequence

$$
0 \longrightarrow \operatorname{Aut}_{\mathbb{P}_{k_{s}}^{n-1}}\left(\mathscr{O}_{\mathbb{P}_{k_{s}}^{n-1}}(1)\right) \longrightarrow \operatorname{Aut}_{k_{s}}\left(\mathbb{P}_{k_{s}}^{n-1}, O_{\mathbb{P}_{k_{s}}^{n-1}}(1)\right) \longrightarrow \operatorname{Aut}_{k_{s}}\left(\mathbb{P}_{k_{s}}^{n-1}\right) \longrightarrow 0 .
$$

(The extension class of this sequence is the opposite of the class of the standard sequence $0 \rightarrow k_{s}^{*} \rightarrow \mathrm{GL}_{n}\left(k_{s}\right) \rightarrow \mathrm{PGL}_{n}\left(k_{s}\right) \rightarrow 0$ which is used to define $\left.\left[Y_{s}\right]\right)$. Let $\tilde{f}: O_{\mathbb{P}_{k s}^{n-1}}(1) \stackrel{\sim}{\longrightarrow} \xi_{s}$ be a relative isomorphism over $f$. In the above notations, a cochain $\widetilde{\psi}$ lifting the cocycle $\psi$ is defined by putting $\widetilde{\psi}(\sigma)=\widetilde{f}^{-1} \widetilde{\sigma}^{-1} \widetilde{f} \widetilde{\sigma}_{0}$ for all $\sigma \in G_{k}$. (Here $\widetilde{\sigma}_{0}: O_{\mathbb{P}_{k_{s}}^{n-1}}(1) \stackrel{\sim}{\longrightarrow} O_{\mathbb{P}_{k_{s}}^{n-1}}(1)$ is the canonical relative isomorphism over $\sigma_{0}: \mathbb{P}_{k_{s}}^{n-1} \stackrel{\sim}{\longrightarrow} \mathbb{P}_{k_{s}}^{n-1}$.) One checks that $d \widetilde{\psi}=\rho^{\prime}$, whence the image of $[\psi]$ by the connecting map for the above sequence equals the class of the extension (4.12) in $\operatorname{Br}(k)$, and this ends the proof.

\section{Comparison with the $l$-adic Abel-Jacobi map $d_{n}^{2}$}

Let $X$ be as in the introduction. Let $l$ be a prime number different from $\operatorname{char}(k)$. We compare the map $\Delta_{n}^{2}: T(X) \rightarrow \operatorname{Ext}_{G_{k}}^{2}\left(H^{2}\left(\bar{X}, \mathbb{G}_{m}\right), \bar{k}^{*}\right) \mathbb{Q}_{\mathbb{Q}}($ see (1.7)) with the $l$-adic Abel-Jacobi map $d_{n}^{2}: T(X) \rightarrow H_{\text {cont }}^{2}\left(G_{k}, H^{2 n-2}\left(\bar{X}, \mathbb{Q}_{l}(n)\right)\right)$ (see (1.9)). To this end, we first relate both target groups.

From the direct sum decomposition $H^{2}\left(\bar{X}, \mathbb{G}_{m}\right)=\bigoplus_{l^{\prime}} H^{2}\left(\bar{X}, \mathbb{G}_{m}\right)\left(l^{\prime}\right)$ with $l^{\prime}$ running over all prime integers, one obtains a product decomposition

$$
\operatorname{Ext}_{G_{k}}^{2}\left(H^{2}\left(\bar{X}, \mathbb{G}_{m}\right), \bar{k}^{*}\right)=\prod_{l^{\prime}} \operatorname{Ext}_{G_{k}}^{2}\left(H^{2}\left(\bar{X}, \mathbb{G}_{m}\right)\left(l^{\prime}\right), \bar{k}^{*}\right) .
$$

We consider the projection onto the $l$-factor

$$
\operatorname{Ext}_{G_{k}}^{2}\left(H^{2}\left(\bar{X}, \mathbb{G}_{m}\right), \bar{k}^{*}\right)_{\mathbb{Q}} \longrightarrow \operatorname{Ext}_{G_{k}}^{2}\left(H^{2}\left(\bar{X}, \mathbb{G}_{m}\right)(l), \bar{k}^{*}\right)_{\mathbb{Q}} .
$$


Next, the Kummer sequence for $\mathbb{G}_{m, \bar{X}}$ gives the exact sequence of discrete $G_{k^{-}}$ modules

$$
0 \longrightarrow \mathrm{NS}(\bar{X}) \otimes \mathbb{Q}_{l} / \mathbb{Z}_{l} \longrightarrow H^{2}\left(\bar{X}, \mathbb{Q}_{l} / \mathbb{Z}_{l}(1)\right) \longrightarrow H^{2}\left(\bar{X}, \mathbb{G}_{m}\right)(l) \longrightarrow 0 .
$$

This sequence is canonically $\mathbb{Q}$-split, at least under the assumption that homological equivalence and numerical equivalence coincide for algebraic 1-cycles on $\bar{X}$ (see [K, page 379]), whence unconditionally for surfaces and varieties in characteristic zero, among others (see [K, page 380]). Namely, if $I_{1}(\bar{X})$ denotes the image of the cycle map $Z_{1}(\bar{X}) \rightarrow H^{2 n-2}\left(\bar{X}, \mathbb{Z}_{l}(n-1)\right)$, the nondegeneracy modulo torsion of the intersection product $I_{1}(\bar{X}) \otimes \mathrm{NS}(\bar{X}) \rightarrow \mathbb{Z}$ implies that the composite map

$$
\begin{aligned}
H^{2}\left(\bar{X}, \mathbb{Q}_{l} / \mathbb{Z}_{l}(1)\right) \stackrel{\sim}{\longrightarrow} \operatorname{Hom}_{\mathbb{Z}_{l}}\left(H^{2 n-2}\left(\bar{X}, \mathbb{Z}_{l}(n-1)\right), \mathbb{Q}_{l} / \mathbb{Z}_{l}\right) \\
\longrightarrow \operatorname{Hom}_{\mathbb{Z}_{l}}\left(I_{1}(\bar{X}) \otimes \mathbb{Z}_{l}, \mathbb{Q}_{l} / \mathbb{Z}_{l}\right)
\end{aligned}
$$

restricts to $\mathrm{NS}(\bar{X}) \otimes \mathbb{Q}_{l} / \mathbb{Z}_{l}$, giving a $\mathbb{Q}$-isomorphism. It follows then that the induced morphism

$$
v: \operatorname{Ext}_{G_{k}}^{2}\left(H^{2}\left(\bar{X}, \mathbb{G}_{m}\right)(l), \bar{k}^{*}\right)_{\mathbb{Q}} \rightarrow \operatorname{Ext}_{G_{k}}^{2}\left(H^{2}\left(\bar{X}, \mathbb{Q}_{l} / \mathbb{Z}_{l}(1)\right), \bar{k}^{*}\right)_{\mathbb{Q}}
$$

is split injective.

On the other hand, we have the tautological isomorphism

$$
H_{\text {cont }}^{2}\left(G_{k}, H^{2 n-2}\left(\bar{X}, \mathbb{Q}_{l}(n)\right)\right) \stackrel{\sim}{\longrightarrow} \operatorname{Ext}_{G_{k}}^{2}\left(\mathbb{Z}_{l}, H^{2 n-2}\left(\bar{X}, \mathbb{Z}_{l}(n)\right)\right)_{\mathbb{Q}} .
$$

Here, the extensions are taken as continuous $\mathbb{Z}_{l}\left[G_{k}\right]$-modules. Then, by Pontryagin duality (twisted by (1)) and Poincaré duality, we have an isomorphism

$$
\operatorname{Ext}_{G_{k}}^{2}\left(\mathbb{Z}_{l}, H^{2 n-2}\left(\bar{X}, \mathbb{Z}_{l}(n)\right)\right)_{\mathbb{Q}} \stackrel{\sim}{\longrightarrow} \operatorname{Ext}_{G_{k}}^{2}\left(H^{2}\left(\bar{X}, \mathbb{Q}_{l} / \mathbb{Z}_{l}(1)\right), \mathbb{Q}_{l} / \mathbb{Z}_{l}(1)\right)_{\mathbb{Q}} .
$$

Here, extensions are taken as discrete ( $l$-primary) torsion $G_{k}$-modules. Next, the obvious map yields an isomorphism

$$
\operatorname{Ext}_{G_{k}}^{2}\left(H^{2}\left(\bar{X}, \mathbb{Q}_{l} / \mathbb{Z}_{l}(1)\right), \mathbb{Q}_{l} / \mathbb{Z}_{l}(1)\right)_{\mathbb{Q}} \stackrel{\sim}{\longrightarrow} \operatorname{Ext}_{G_{k}}^{2}\left(H^{2}\left(\bar{X}, \mathbb{Q}_{l} / \mathbb{Z}_{l}(1)\right), \bar{k}^{*}\right)_{\mathbb{Q}} .
$$

This map factors through $\operatorname{Ext}_{G_{k}}^{2}\left(H^{2}\left(\bar{X}, \mathbb{Q}_{l} / \mathbb{Z}_{l}(1)\right), \bar{k}^{*}(l)\right)_{\mathbb{Q}}$, where the extensions are taken here as discrete $G_{k}$-modules. We show that both of the factor maps are isomorphisms. The isomorphism

$$
\operatorname{Ext}_{G_{k}}^{2}\left(H^{2}\left(\bar{X}, \mathbb{Q}_{l} / \mathbb{Z}_{l}(1)\right), \mathbb{Q}_{l} / \mathbb{Z}_{l}(1)\right)_{\mathbb{Q}} \stackrel{\sim}{\longrightarrow} \operatorname{Ext}_{G_{k}}^{2}\left(H^{2}\left(\bar{X}, \mathbb{Q}_{l} / \mathbb{Z}_{l}(1)\right), \bar{k}^{*}(l)\right)_{\mathbb{Q}}
$$

is a consequence of the following more general statement. Let $\bar{M}, \bar{N}$ be torsion discrete $G_{k}$-modules. Then, for all $i \geq 0, \operatorname{Ext}_{G_{k}}^{i}(\bar{M}, \bar{N})^{\prime} \stackrel{\sim}{\longrightarrow} \operatorname{Ext}_{G_{k}}^{i}(\bar{M}, \bar{N})$, where the first group classifies extensions of torsion discrete $G_{k}$-modules and the second 
one classifies extensions of discrete $G_{k}$-modules. In fact, we may construct an injective resolution $\bar{I}$ of $\bar{N}$ made out of torsion modules; whence $\operatorname{Ext}_{G_{k}}^{i}(\bar{M}, \bar{N})^{\prime}=$ $\operatorname{Hom}_{G_{k}}\left(\bar{M}, \bar{I}^{\cdot}\right)=\operatorname{Ext}_{G_{k}}^{i}(\bar{M}, \bar{N})$. To obtain such a resolution, one can choose an embedding $\bar{N} \hookrightarrow \bar{N}^{\prime}$ into a divisible torsion abelian group; take $\bar{I}^{0}=\operatorname{Maps}_{\text {cont }}\left(G_{k}, \bar{N}^{\prime}\right)$, and then proceed inductively (see [S, page I-12], or [Mi1, Chapter III, Remark 1.4(a)]). As for the isomorphism

$$
\operatorname{Ext}_{G_{k}}^{2}\left(H^{2}\left(\bar{X}, \mathbb{Q}_{l} / \mathbb{Z}_{l}(1)\right), \bar{k}^{*}(l)\right)_{\mathbb{Q}} \stackrel{\sim}{\longrightarrow} \operatorname{Ext}_{G_{k}}^{2}\left(H^{2}\left(\bar{X}, \mathbb{Q}_{l} / \mathbb{Z}_{l}(1)\right), \bar{k}^{*}\right)_{\mathbb{Q}},
$$

this follows from the vanishing of the groups $\operatorname{Ext}_{G_{k}}^{i}\left(H^{2}\left(\bar{X}, \mathbb{Q}_{l} / \mathbb{Z}_{l}(1)\right), \bar{k}^{*} / \bar{k}^{*}(l)\right)$. (One can use, for instance, [Mi2, Chapter I, Corollary 0.23].)

\section{THEOREM 5.1}

Let $X$ be, as above, a smooth, projective, geometrically irreducible variety of dimension $n$ over a field $k$. Let l be a prime integer different from $\operatorname{char}(k)$. Then the following diagram is commutative:

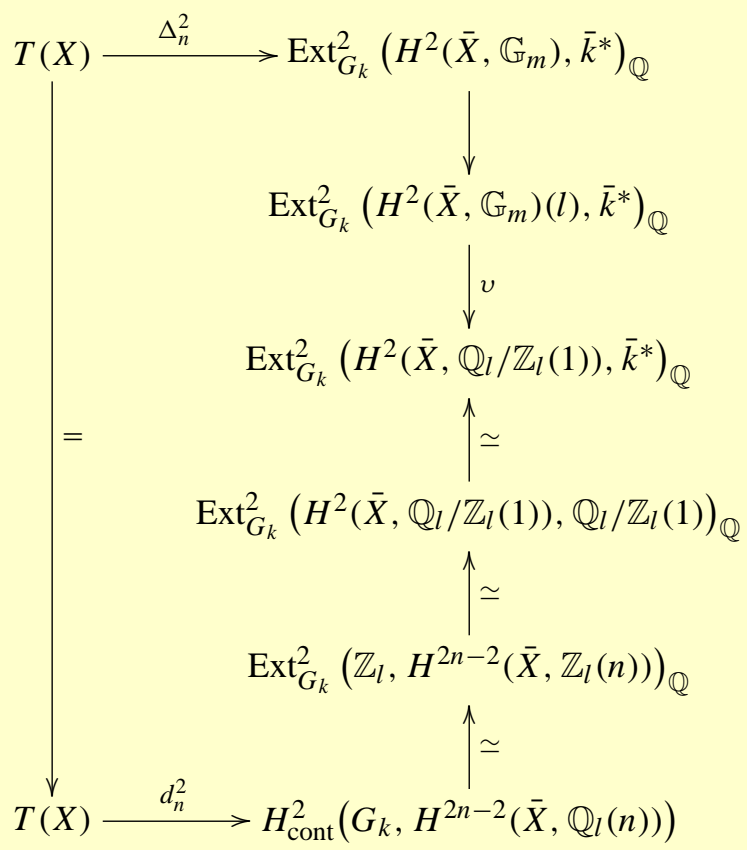

Moreover, if the conjectured equality of homological and numerical equivalence holds for algebraic 1-cycles on $\bar{X}$ (e.g., if $n=2)$, then the map $v$ is injective.

\section{Proof}

The last part has been already discussed. As for the first part, let $z$ be a 0 -cycle of 
degree zero on $X$, such that $\Delta_{n}^{1}([z])=0$. Choose $Z \subset X$ a 0 -dimensional closed subset containing the support $|z|$ of $z$. Furthermore, let $\bar{Z} \subset \bar{X}$ be as in Section 2. We choose a morphism $\tilde{z}$ as in (3.1) and also call $\tilde{z}$ its restriction to the $l$-primary torsion parts (see Section 2)

$$
H_{c}^{1}\left(\bar{X} \backslash \bar{Z}, \mathbb{Q}_{l} / \mathbb{Z}_{l}(1)\right)=\operatorname{Pic}(\bar{X}, \bar{Z})(l) \stackrel{\tilde{z}}{\longrightarrow} \bar{k}^{*}(l)=\mathbb{Q}_{l} / \mathbb{Z}_{l}(1) .
$$

On the other hand, let $\mathscr{I}$ be an injective resolution of $\mathbb{G}_{m, X, Z}$. As this sheaf is $l$ divisible, it follows that, for all $r \in \mathbb{N}, l^{r} \mathscr{I}$ is an injective resolution of $\mu l^{r}, X, Z=$ ${ }_{l}^{r} \mathbb{G}_{m, X, Z}$ as a $\left(\mathbb{Z} / l^{r} \mathbb{Z}\right)$-module. This is the sheaf $j ! \mu_{l^{r}, X \backslash Z}$, where the letter $j$ denotes the inclusion $X \backslash Z \hookrightarrow X$. Put $\mathscr{I} \cdot(l)=\bigcup_{r \in \mathbb{N}}\left(l^{r} \mathscr{I}\right) \subset \mathscr{I}$. Keeping the notation from Section 4, the morphism $h_{*} \mathscr{I}^{\cdot}(l) \rightarrow h_{*} \mathscr{I}$ induces a map $\chi_{1}\left(h_{*} \mathscr{I}^{\cdot}(l)\right) \rightarrow$ $\chi_{1}\left(h_{*} \mathscr{I}^{\cdot}\right) \rightarrow \chi_{1}\left(h_{*} \mathscr{I}^{\cdot}\right)_{R}$, which we represent as

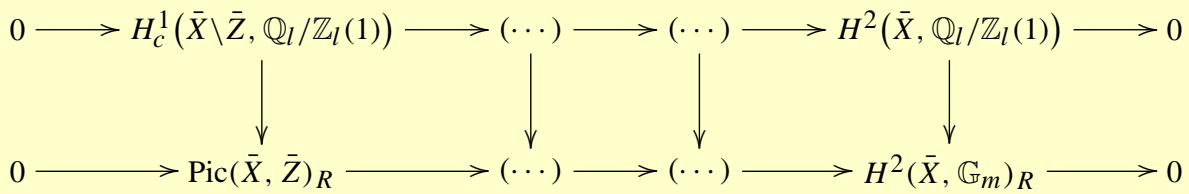

(Here we use the equality $H_{c}^{2}\left(\bar{X} \backslash \bar{Z}, \mathbb{Q}_{l} / \mathbb{Z}_{l}(1)\right)=H^{2}\left(\bar{X}, \mathbb{Q}_{l} / \mathbb{Z}_{l}(1)\right)$.) By Proposition (4.10), the bottom row in (5.3) represents $-\left(\alpha_{Z}\right)_{R} \in \operatorname{Ext}_{G_{k}}^{2}\left(H^{2}\left(\bar{X}, \mathbb{G}_{m}\right)_{R}\right.$, $\left.\operatorname{Pic}(\bar{X}, \bar{Z})_{R}\right)$. Taking pushouts in (5.3) by $\tilde{z}: H_{c}^{1}\left(\bar{X} \backslash \bar{Z}, \mathbb{Q}_{l} / \mathbb{Z}_{l}(1)\right) \rightarrow \mathbb{Q}_{l} / \mathbb{Z}_{l}(1)$ (top row) and $\widetilde{z}_{R}: \operatorname{Pic}(\bar{X}, \bar{Z})_{R} \rightarrow \bar{k}_{R}^{*}=\bar{k}^{*}$ (bottom row), respectively, we obtain an exact commutative diagram

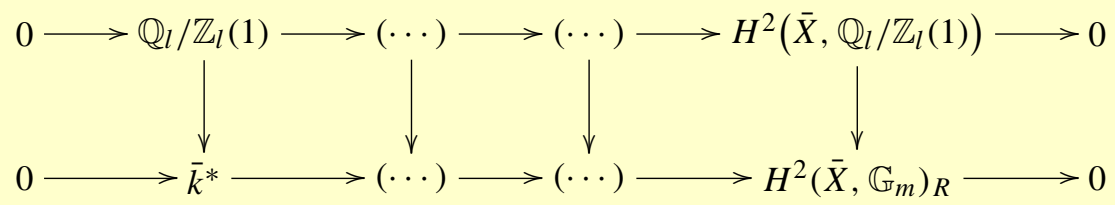

implying that the image of $[z]$ in $\operatorname{Ext}_{G_{k}}^{2}\left(H^{2}\left(\bar{X}, \mathbb{Q}_{l} / \mathbb{Z}_{l}(1)\right), \bar{k}^{*}\right)_{\mathbb{Q}}$ by $\Delta_{n}^{2}$ is the opposite of the image of the element of $\operatorname{Ext}_{G_{k}}^{2}\left(H^{2}\left(\bar{X}, \mathbb{Q}_{l} / \mathbb{Z}_{l}(1)\right), \mathbb{Q}_{l} / \mathbb{Z}_{l}(1)\right)_{\mathbb{Q}}$ represented by the top row in (5.4). Thus it is sufficient to show that the latter element equals the opposite of the image of $[z]$ by $d_{n}^{2}$. Now, the (twisted) Pontryagin dual of the top row in (5.4) is obtained from the dual of the top row in (5.3) by taking pullback via the dual map of (5.2), $\tilde{z}: \mathbb{Z}_{l} \rightarrow H^{2 n-1}\left(\bar{X} \backslash \bar{Z}, \mathbb{Z}_{l}(n)\right)$. By Poincare duality, we have, writing for a moment $h^{\prime}: X \backslash Z \rightarrow \operatorname{Spec}(k)$ for the restriction of $h: X \rightarrow \operatorname{Spec}(k)$,

$$
\left(\mathrm{Rh}_{*}^{\prime} \mathbb{Z}_{l}(n)_{X \backslash Z}\right)[2 n] \cong \operatorname{Hom}_{\mathbb{Z}}\left(\operatorname{Rh}_{*}\left(j ! \mathbb{Q}_{l} / \mathbb{Z}_{l}(1)_{X \backslash Z}\right), \mathbb{Q}_{l} / \mathbb{Z}_{l}(1)\right)
$$


Hence $\operatorname{Hom}\left(\chi_{1}\left(h_{*} \mathscr{I}^{\cdot}(l)\right), \mathbb{Q}_{l} / \mathbb{Z}_{l}(1)\right)=\chi_{-2}\left(\operatorname{Hom}_{\mathbb{Z}}\left(h_{*} \mathscr{I} \cdot(l), \mathbb{Q}_{l} / \mathbb{Z}_{l}(1)\right)\right)$ represents the class $\chi_{2 n-2}\left(\mathrm{Rh}_{*}^{\prime} \mathbb{Z}_{l}(n)_{X \backslash Z}\right)$. The desired conclusion then follows from [J4, Theorem 1, p. 263].

\section{Remark 5.5}

(a) Suppose that $\operatorname{char}(k)=p>0$. Then, since $\bar{k}^{*}$ is uniquely $p$-divisible, it follows from [Mi2, Chapter I, Corollary 0.23] that $\operatorname{Ext}_{G_{k}}^{i}\left(H^{2}\left(\bar{X}, \mathbb{G}_{m}\right)(p), \bar{k}^{*}\right)=0$ for all $i \geq 0$. Thus $\operatorname{Ext}_{G_{k}}^{2}\left(H^{2}\left(\bar{X}, \mathbb{G}_{m}\right), \bar{k}^{*}\right)=\operatorname{Ext}_{G_{k}}^{2}\left(H^{2}\left(\bar{X}, \mathbb{G}_{m}\right)(n o p), \bar{k}^{*}\right)$ in this case. Theorem (5.1) implies therefore that, modulo the assumption on the algebraic 1cycles of $\bar{X}$, the datum of $\Delta_{n}^{2}$ is equivalent to the set of data of all $d_{n}^{2}$ for $l \neq p$. In particular, and with this caveat, Proposition 3.17 is also a consequence of (the proof of) Theorem 5.1.

(b) Thus working with the algebraic closure $\bar{k}$ of $k$ in the definition of (1.7) has the effect of stripping off a possible contribution of the $p$-part of the group $H^{2}\left(\bar{X}, \mathbb{G}_{m}\right)$. Now, the whole theory can be developed equally with $\bar{k}$ and $\bar{X}$ replaced by $k_{s}$ and $X_{s}=X \otimes_{k} k_{s}$, respectively, leading to a map

$$
T(X)=F^{2} \mathrm{CH}_{0}(X) \stackrel{\Delta_{n}^{2}}{\longrightarrow} \operatorname{Ext}_{G_{k}}^{2}\left(H^{2}\left(X_{s}, \mathbb{G}_{m}\right), k_{s}^{*}\right)_{\mathbb{Q}} .
$$

(We use this in Section 7.) One has, canonically,

$$
\begin{aligned}
\operatorname{Ext}_{G_{k}}^{2}\left(H^{2}\left(X_{s}, \mathbb{G}_{m}\right), k_{s}^{*}\right) \cong & \operatorname{Ext}_{G_{k}}^{2}\left(H^{2}\left(\bar{X}, \mathbb{G}_{m}\right), \bar{k}^{*}\right) \\
& \oplus \operatorname{Ext}_{G_{k}}^{2}\left(H^{2}\left(X_{s}, \mathbb{G}_{m}\right)(p), k_{s}^{*}\right),
\end{aligned}
$$

and the map (5.6) induces, in particular, our former map (1.7) by projection. There remains then an as yet unexplored component along the $p$-part of the group $H^{2}\left(X_{s}, \mathbb{G}_{m}\right)$. However, this does not seem to be the right approach since, for $k$ a perfect field, we do not obtain anything new from it. Instead, developing the missing $p$-part of this theory when $\operatorname{char}(k)=p>0$ possibly involves dealing with extensions of geometrical objects rather than extensions of Galois modules. This would provide a further step towards a hypothetical group object of (hyper)geometrical nature, allowing us to parallel for (1.7) the passage from (1.3) and (1.4) to (1.5) and (1.6), respectively.

(c) In a vein similar to that of (b), for all $i \geq 0$, the canonical isomorphisms

$$
\operatorname{Ext}_{G_{k}}^{i}\left(H^{i}\left(\bar{X}, \mathbb{G}_{m}\right), \bar{k}^{*}\right) \stackrel{\sim}{\longrightarrow} \operatorname{Ext}_{G_{k}}^{i}\left(H^{i}\left(X_{s}, \mathbb{G}_{m}\right), \bar{k}^{*}\right)
$$

yield natural maps

$$
\operatorname{Ext}_{G_{k}}^{i}\left(H^{i}\left(X_{s}, \mathbb{G}_{m}\right), k_{s}^{*}\right) \longrightarrow \operatorname{Ext}_{G_{k}}^{i}\left(H^{i}\left(\bar{X}, \mathbb{G}_{m}\right), \bar{k}^{*}\right) .
$$


For $i=1$, the analogous construction to that for $\Delta_{n}^{1}$ (see Section 3) with $\bar{k}$ and $\bar{X}$ replaced, respectively, by $k_{s}$ and $X_{s}$, leads to a map

$$
A_{0}(X)=F^{1} \mathrm{CH}_{0}(X) \stackrel{\Delta_{n}^{1}}{\longrightarrow} \operatorname{Ext}_{G_{k}}^{1}\left(H^{1}\left(X_{s}, \mathbb{G}_{m}\right), k_{s}^{*}\right)
$$

which lifts (1.4) (we use this in Section 7). Once more, this is not very illuminating, since both maps stem here from a common, well understood, more powerful geometrical construction (see Section 1).

\section{A comparison with M. Green's method}

In this section we deal with surfaces only. We use our construction to relate the AbelJacobi map $d_{2}^{2}$ to M. Green's construction in [G]. (Well understood, M. Green works in the setting of complex algebraic varieties, with (arithmetic) mixed Hodge structures, while we are working here in the abstract setting with $l$-adic theory). For 0cycles on a fixed curve on a smooth, projective, geometrically irreducible surface $X$ over $k$, the 2-extension class provided by the higher Abel-Jacobi map $d_{2}^{2}$ is described as the composition of two 1-extensions, one of them reflecting the relation of the 0 cycle with its curve environment and the second one relating the curve to the surface. As a matter of fact, we consider here only the simplest case, that of a smooth, geometrically irreducible (projective) curve $C \subset X$ over $k$. Let $z$ be a 0-cycle of degree zero on $X$, such that $|z| \subset C$ and such that $[z] \in T(X)$. We have a pair of dual morphisms of abelian varieties over $k, \underline{\mathrm{Pic}}_{X / k}^{0} \rightarrow \underline{\mathrm{Pic}}_{C / k}^{0}$ and $J(C) \rightarrow \mathrm{Alb}(X)$, which we complete to exact sequences

$$
\begin{aligned}
& 0 \longrightarrow P \longrightarrow J(C) \longrightarrow \operatorname{Alb}(X) \longrightarrow \widehat{R}^{0} \longrightarrow 0 \\
& 0 \longrightarrow R \longrightarrow \underline{\mathrm{Pic}}_{X / k}^{0} \longrightarrow \underline{\mathrm{Pic}}_{C / k}^{0} \longrightarrow \widehat{P}^{0} \longrightarrow 0 .
\end{aligned}
$$

Here $P$ and $R$ are algebraic groups, $P^{0}$ and $R^{0}$ are the respective identity components, and $\widehat{P}^{0}$ and $\widehat{R}^{0}$ are the duals of these abelian varieties. The 0 -cycle $z$ determines an element of $P(k)$, whence an element of $P(k)_{\mathbb{Q}}=P^{0}(k)_{\mathbb{Q}}$. By duality, this yields an element of $\operatorname{Ext}^{1}\left(\widehat{P}^{0}, \mathbb{G}_{m, k}\right) \mathbb{Q}$ (extensions of commutative group schemes over $k$ ), whence an element of $\operatorname{Ext}_{G_{k}}^{1}\left(\widehat{\bar{P}}^{0}, \bar{k}^{*}\right)_{\mathbb{Q}}$. We represent this as

$$
0 \longrightarrow \bar{k}^{*} \longrightarrow(\cdots) \longrightarrow \widehat{\bar{P}}^{0} \longrightarrow 0 .
$$

We fix a prime number $l$, different from the characteristic of $k$. By taking the pullback of $(6.3)$ by $\widehat{\bar{P}}^{0}(l) \hookrightarrow \widehat{\bar{P}}^{0}$, we derive an extension

$$
0 \longrightarrow \bar{k}^{*} \longrightarrow(\cdots) \longrightarrow \widehat{\bar{P}}^{0}(l) \longrightarrow 0 .
$$

On the other hand, the inductive limit of the cohomology sequences of the (selfdefining) exact sequences on $\bar{X}$,

$$
0 \longrightarrow \mu_{l^{r}, \bar{X}, \bar{C}} \longrightarrow \mu_{l^{r}, \bar{X}} \longrightarrow \mu_{l^{r}, \bar{C}} \longrightarrow 0
$$


gives

$$
\operatorname{Pic}(\bar{X})(l) \longrightarrow \operatorname{Pic}(\bar{C})(l) \longrightarrow H_{c}^{2}\left(\bar{X} \backslash \bar{C}, \mathbb{Q}_{l} / \mathbb{Z}_{l}(1)\right) \longrightarrow\langle[\bar{C}]\rangle^{\perp} \longrightarrow 0 .
$$

Here $\langle[\bar{C}]\rangle^{\perp} \subset H^{2}\left(\bar{X}, \mathbb{Q}_{l} / \mathbb{Z}_{l}(1)\right)$ is the orthogonal submodule of $\langle[\bar{C}]\rangle \subset$ $H^{2}\left(\bar{X}, \mathbb{Z}_{l}(1)\right)$. From $(6.2)$ we deduce a natural map $\operatorname{NS}(\bar{X})(l) \rightarrow \widehat{\widehat{P}}^{0}(l)$, and the above sequence yields

$$
0 \longrightarrow \widehat{\bar{P}}^{0}(l) / \operatorname{Im}(\mathrm{NS}(\bar{X})(l)) \longrightarrow H_{c}^{2}\left(\bar{X} \backslash \bar{C}, \mathbb{Q}_{l} / \mathbb{Z}_{l}(1)\right) \longrightarrow\langle[\bar{C}]\rangle^{\perp} \longrightarrow 0 .
$$

If $l$ does not divide $\mid$ Tors $(\operatorname{NS}(\bar{X})) \mid$, then the first term in $(6.5)$ becomes $\widehat{\bar{P}}^{0}(l)$. In any case, we have a $\mathbb{Q}$-exact sequence

$$
0 \longrightarrow \widehat{\bar{P}}^{0}(l) \longrightarrow H_{c}^{2}\left(\bar{X} \backslash \bar{C}, \mathbb{Q}_{l} / \mathbb{Z}_{l}(1)\right) \longrightarrow\langle[\bar{C}]\rangle^{\perp} \longrightarrow 0
$$

in the sense that it is exact in the $\mathbb{Q}$-localized category of the category of discrete $G_{k}$-modules. We have, moreover, $\left(\operatorname{NS}(\bar{X}) \otimes \mathbb{Z}_{l}\right)^{\perp} \subset\langle[\bar{C}]\rangle^{\perp}$ and (see Section 5) a $\mathbb{Q}$-isomorphism $\left(\operatorname{NS}(\bar{X}) \otimes \mathbb{Z}_{l}\right)^{\perp} \stackrel{\sim}{\longrightarrow} \operatorname{Br}(\bar{X})(l)$. This is a true isomorphism if $l$ does not divide $\mid$ Tors $\mathrm{NS}(\bar{X}) \mid$ or the discriminant of the intersection form $\mathrm{NS}(\bar{X})_{0} \otimes$ $\mathrm{NS}(\bar{X})_{0} \rightarrow \mathbb{Z}$ (with $\mathrm{NS}(\bar{X})_{0}$ standing for $\mathrm{NS}(\bar{X}) /$ Tors $\mathrm{NS}(\bar{X})$ ). By pulling back (6.6) by the morphism $\operatorname{Br}(\bar{X})(l) \rightarrow\left(\operatorname{NS}(\bar{X}) \otimes \mathbb{Z}_{l}\right)^{\perp} \hookrightarrow\langle[\bar{C}]\rangle^{\perp}$, we obtain a $\mathbb{Q}$-extension

$$
0 \longrightarrow \widehat{\bar{P}}^{0}(l) \longrightarrow(\cdots) \longrightarrow \operatorname{Br}(\bar{X})(l) \longrightarrow 0 .
$$

\section{THEOREM 6.8}

Let the assumptions be as at the beginning of Section 6. In the above notation, the image of $\Delta_{2}^{2}([z])$ in $\operatorname{Ext}_{G_{k}}^{2}\left(\operatorname{Br}(\bar{X})(l), \bar{k}^{*}\right)_{\mathbb{Q}}$ is represented by the composition of the 1-extensions (6.4) and (6.7).

\section{Proof}

Let $\bar{S}$ be a finite set of closed, irreducible curves of $\bar{X}$ adequate for $Z=|z|$, containing the curve $\bar{C}$, and such that, furthermore, the classes $\left[\bar{C}^{\prime}\right], \bar{C}^{\prime} \in \bar{S}$, generate the group NS $(\bar{X})$. The morphisms of two-term complexes of sheaves on $\bar{X}$,

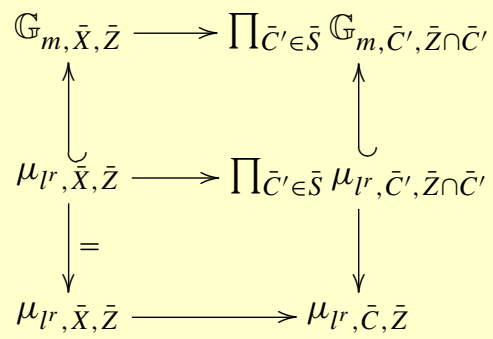


yield, after taking inductive limits, morphisms of exact sequences
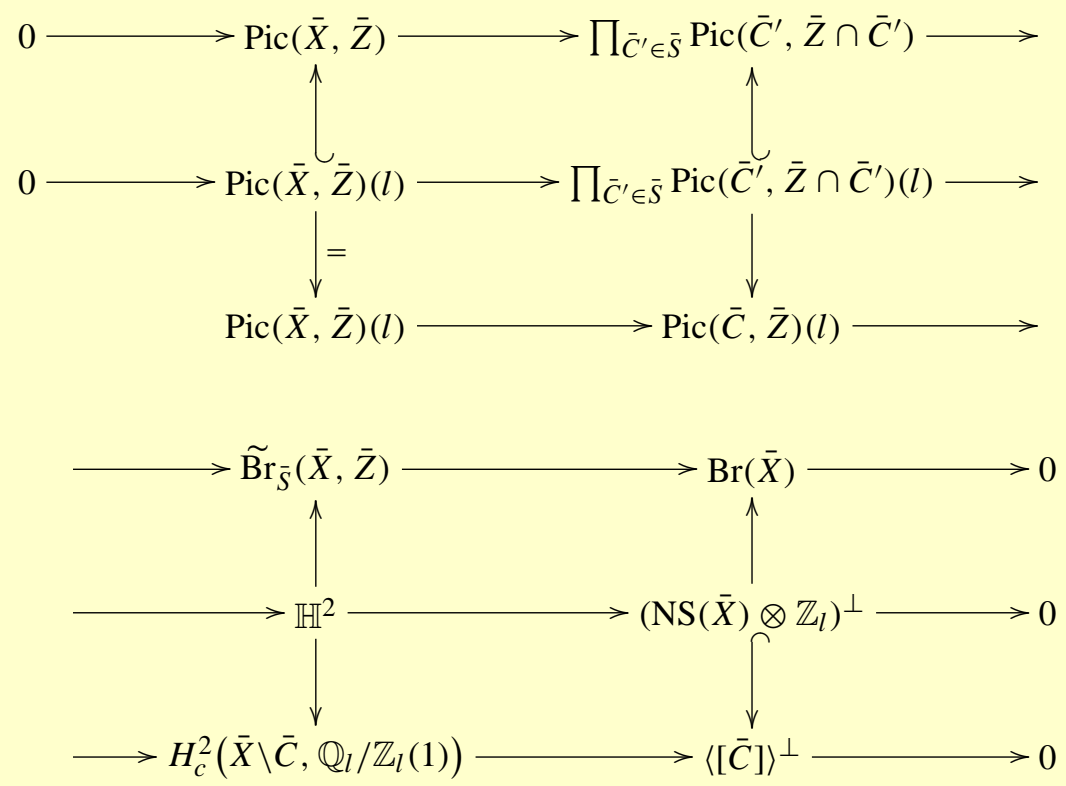

The upper outer right vertical map induces the $\mathbb{Q}$-isomorphism $\left(\mathrm{NS}(\bar{X}) \otimes \mathbb{Z}_{l}\right)^{\perp} \rightarrow$ $\operatorname{Br}(\bar{X})(l)$ alluded to above. Therefore, if $\widetilde{z}: \operatorname{Pic}(\bar{X}, \bar{Z}) \rightarrow \bar{k}^{*}$ is a morphism as in (3.1), then pushing out the middle row by the restriction of $\widetilde{z}$ to $\operatorname{Pic}(\bar{X}, \bar{Z})(l)$ and pulling back the resulting sequence by the morphism $\operatorname{Br}(\bar{X})(l) \rightarrow\left(\mathrm{NS}(\bar{X}) \otimes \mathbb{Z}_{l}\right)^{\perp}$ yields a representative of the image of $\Delta_{2}^{2}([z])$ in $\operatorname{Ext}_{G_{k}}^{2}\left(\operatorname{Br}(\bar{X})(l), \bar{k}^{*}\right)_{\mathbb{Q}}$. On the other hand, the right-hand-side half of the bottom row contains the exact sequence (6.5) (or (6.6) in terms of $\mathbb{Q}$-extensions). Therefore it suffices to show that $\widetilde{z}$ can be chosen so as to provide a commutative diagram

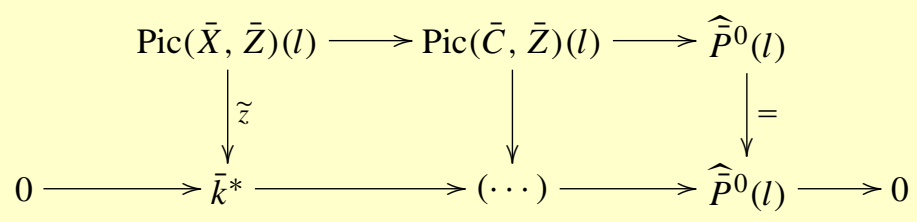

where the bottom row is (6.4).

To settle this point, we introduce $\operatorname{Pic}^{0}(\bar{X}, \bar{Z}) \subset \operatorname{Pic}(\bar{X}, \bar{Z})$ and $\operatorname{Pic}^{0}(\bar{C}, \bar{Z}) \subset$ $\operatorname{Pic}(\bar{C}, \bar{Z})$ as the inverse images of $\operatorname{Pic}^{0}(\bar{X}) \subset \operatorname{Pic}(\bar{X})$ and $\operatorname{Pic}^{0}(\bar{C}) \subset \operatorname{Pic}(\bar{C})$, respectively. We deduce from (6.2) an exact sequence

$$
0 \longrightarrow \bar{R} \longrightarrow \operatorname{Pic}^{0}(\bar{X}, \bar{Z}) \longrightarrow \operatorname{Pic}^{0}(\bar{C}, \bar{Z}) \longrightarrow \widehat{\bar{P}}^{0} \longrightarrow 0 .
$$


On the other hand, we have a commutative diagram with exact rows

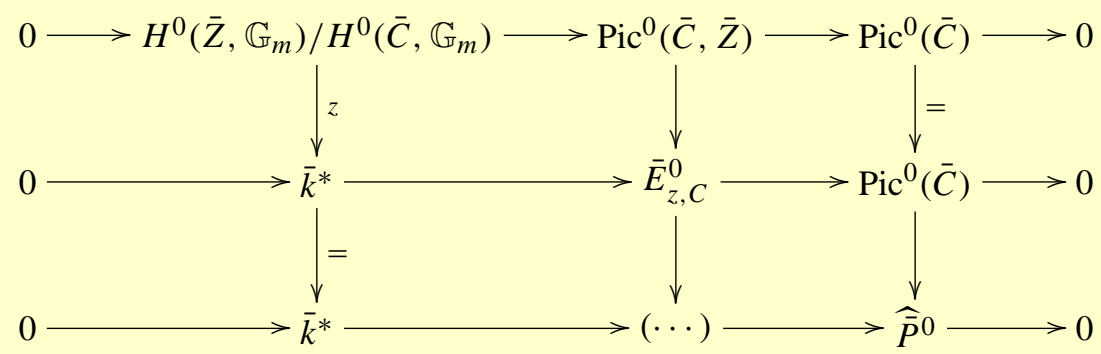

where the bottom row is $(6.3)$ and the middle row is the restriction to $\operatorname{Pic}^{0}(\bar{C})$ of the extension representing $\Delta_{1, C}^{1}([z])$. From this diagram we deduce the following one:

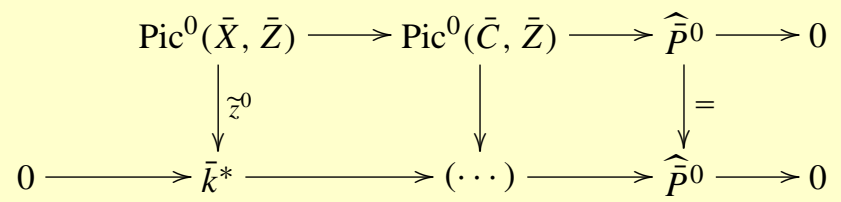

with the morphism $\widetilde{z}^{0}$ extending the map $z: H^{0}\left(\bar{Z}, \mathbb{G}_{m}\right) / H^{0}\left(\bar{X}, \mathbb{G}_{m}\right) \rightarrow \bar{k}^{*}$. By the exact sequence

$$
0 \longrightarrow \operatorname{Pic}^{0}(\bar{X}, \bar{Z}) \longrightarrow \operatorname{Pic}(\bar{X}, \bar{Z}) \longrightarrow \mathrm{NS}(\bar{X}) \longrightarrow 0
$$

and Remark (3.9), we may assume that $\widetilde{z}^{0}$ is the restriction of a morphism $\widetilde{z}$ : $\operatorname{Pic}(\bar{X}, \bar{Z}) \rightarrow \bar{k}^{*}$ as in (3.1). From this we deduce at once a diagram like (6.9), taking into account that $\operatorname{Pic}^{0}(\bar{C}, \bar{Z})(l)=\operatorname{Pic}(\bar{C}, \bar{Z})(l)$ and that, by the exact sequence

$$
0 \longrightarrow \operatorname{Pic}^{0}(\bar{X}, \bar{Z})(l) \longrightarrow \operatorname{Pic}(\bar{X}, \bar{Z})(l) \longrightarrow \operatorname{NS}(\bar{X})(l) \longrightarrow 0,
$$

the objects $\operatorname{Pic}^{0}(\bar{X}, \bar{Z})(l)$ and $\operatorname{Pic}(\bar{X}, \bar{Z})(l)$ are $\mathbb{Q}$-isomorphic. This ends the proof of Theorem (6.8).

To end this section we translate this result by means of Pontryagin duality. Notation and assumptions are as at the beginning of Section 6. Taking (twisted) Pontryagin duals in the sequence (6.5) — and using Poincaré duality as well as the duality between the abelian varieties $P^{0}$ and $\widehat{P}^{0}$-we obtain the middle row of the following exact 
diagram:

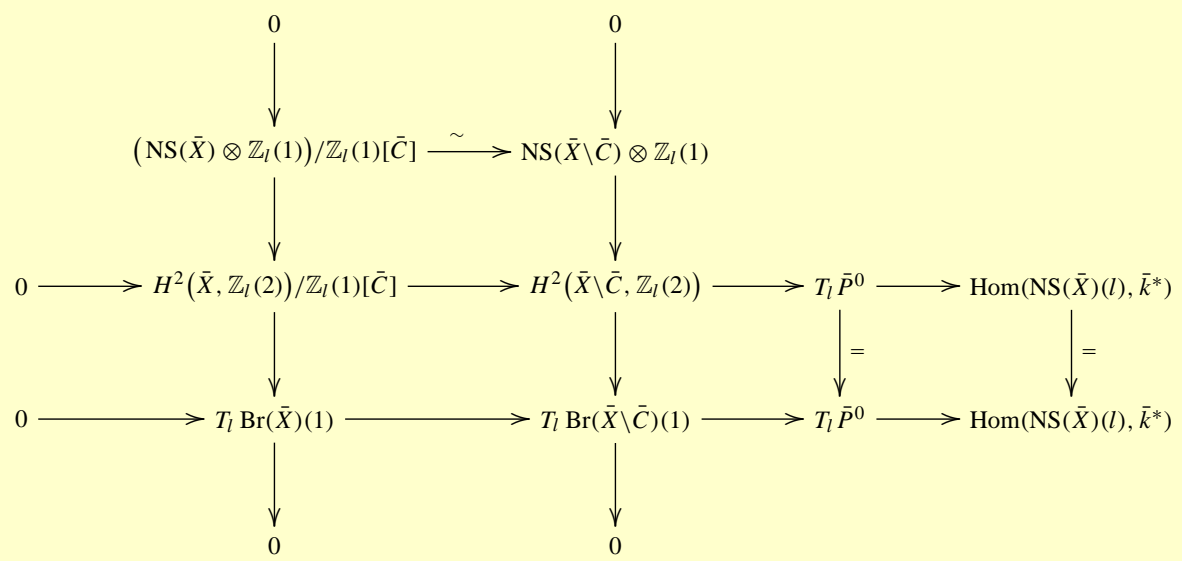

By taking the tensor product with $\mathbb{Q}$, the last row gives the exact sequence of continuous $\mathbb{Q}_{l}\left[G_{k}\right]$-modules

$$
0 \longrightarrow V_{l} \operatorname{Br}(\bar{X})(1) \longrightarrow V_{l} \operatorname{Br}(\bar{X} \backslash \bar{C})(1) \longrightarrow V_{l} \bar{P}^{0} \longrightarrow 0 .
$$

This is the dual counterpart of the $\mathbb{Q}$-extension (6.7). We write

$$
H^{1}\left(G_{k}, V_{l} \bar{P}^{0}\right)=H_{\text {cont }}^{1}\left(G_{k}, V_{l} \bar{P}^{0}\right) \stackrel{\delta}{\longrightarrow} H_{\text {cont }}^{2}\left(G_{k}, V_{l} \operatorname{Br}(\bar{X})(1)\right)
$$

for the first connecting homomorphism of its cohomology sequence. On the other hand, the Kummer sequence for $P^{0}$ gives a morphism $P^{0}(k) \rightarrow H^{1}\left(G_{k}, T_{l} \bar{P}^{0}\right)$, whence also a morphism

$$
P(k)_{\mathbb{Q}}=P^{0}(k)_{\mathbb{Q}} \longrightarrow H^{1}\left(G_{k}, V_{l} \bar{P}^{0}\right) .
$$

One has a commutative diagram

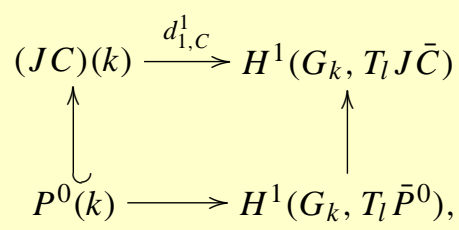

the right-hand-side vertical map being injective if, for example, $k$ is finitely generated over the prime field (by the Mordell-Weil theorem applied to the quotient abelian variety $J C / P$ ). Finally (see Section 5), the canonical splitting of the sequence

$$
0 \longrightarrow \mathrm{NS}(\bar{X}) \otimes \mathbb{Q}_{l}(1) \longrightarrow H^{2}\left(\bar{X}, \mathbb{Q}_{l}(2)\right) \longrightarrow V_{l} \operatorname{Br}(\bar{X})(1) \longrightarrow 0
$$


yields a canonical direct sum decomposition

$$
H^{2}\left(G_{k}, H^{2}\left(\bar{X}, \mathbb{Q}_{l}(2)\right)\right)=H^{2}\left(G_{k}, \operatorname{NS}(\bar{X}) \otimes \mathbb{Q}_{l}(1)\right) \oplus H^{2}\left(G_{k}, V_{l} \operatorname{Br}(\bar{X})(1)\right) .
$$

With the aid of Theorem 5.1, the following is then a restatement of Theorem 6.8.

COROLLARY 6.15

Let $C \subset X$ be a curve on a surface, both $C$ and $X$ smooth, projective, and geometrically irreducible over a field $k$. In the preceding notation, the following diagram is commutative:

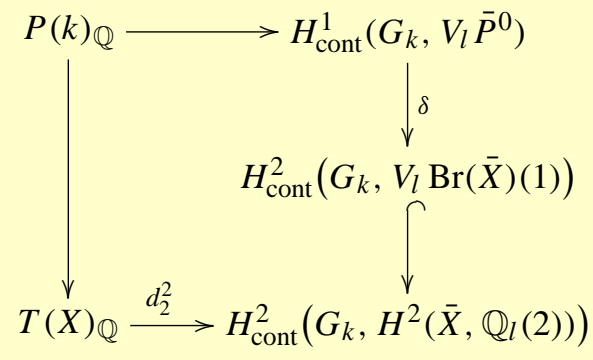

\section{Relation with the pairing between $\mathrm{CH}_{0}(X)$ and $\operatorname{Br}(X)$}

Let $X$ again be as in the introduction. As earlier in this paper, $k_{s}$ denotes a separable algebraic closure of $k$, and $X_{s}=X \otimes_{k} k_{s}$. We consider the pairing

$$
\mathrm{CH}_{0}(X) \otimes_{\mathbb{Z}} \mathrm{Br}(X) \longrightarrow \mathrm{Br}(k)
$$

quoted in Section 1. Recall (see [L], [M]) that, given $[z] \in \mathrm{CH}_{0}(X), z=\sum_{i=1}^{r} n_{i} x_{i}$, and $[P] \in \operatorname{Br}(X)$, the image of $[z] \otimes_{\mathbb{Z}}[P]$ in $\operatorname{Br}(k)$ is given by

$$
\langle[z],[P]\rangle=\sum_{i=1}^{r} n_{i} N_{k\left(x_{i}\right) / k}\left(\left[P_{x_{i}}\right]\right)
$$

with $N_{k\left(x_{i}\right) / k}: \operatorname{Br}\left(k\left(x_{i}\right)\right) \rightarrow \operatorname{Br}(k)$ the norm map.

We write, as before, $F^{0} \mathrm{CH}_{0}(X)=\mathrm{CH}_{0}(X), F^{1} \mathrm{CH}_{0}(X)=A_{0}(X) \subset \mathrm{CH}_{0}(X)$, the set of rational equivalence classes of 0-cycles of degree zero, and $F^{2} \mathrm{CH}_{0}(X)=$ $T(X) \subset A_{0}(X)$, the kernel of the Albanese map $A_{0}(X) \rightarrow \operatorname{Alb}(X)$. On the other hand, the Lyndon-Hochschild-Serre spectral sequence $H^{i}\left(G_{k}, H^{j}\left(X_{s}, \mathbb{G}_{m}\right)\right) \Rightarrow$ $H^{i+j}\left(X, \mathbb{G}_{m}\right)$ yields, in particular, a length 2 filtration $F^{\cdot} \operatorname{Br}(X)$ of $\operatorname{Br}(X)$. We have, canonically, injective maps

$$
\begin{array}{r}
\operatorname{Br}(X) / F^{1} \operatorname{Br}(X) \hookrightarrow H^{0}\left(G_{k}, \operatorname{Br}\left(X_{s}\right)\right), \\
F^{1} \operatorname{Br}(X) / F^{2} \operatorname{Br}(X) \hookrightarrow H^{1}\left(G_{k}, \operatorname{Pic}\left(X_{s}\right)\right),
\end{array}
$$


and $F^{2} \operatorname{Br}(X) \subset \operatorname{Br}(X)$ is the image of $\operatorname{Br}(k)$ by the structure map $X \rightarrow \operatorname{Spec}(k)$. We consider the pairings induced by (7.1):

$$
F^{i} \mathrm{CH}_{0}(X) \otimes_{\mathbb{Z}} F^{2-i} \operatorname{Br}(X) \longrightarrow \operatorname{Br}(k) .
$$

For $i=0$ this is, roughly expressed, multiplication by the degree of the first factor. For $i=1$ this should be, at worst up to a sign, the tensor product of the maps (5.7) and (7.4) followed by the cup product map. We have not been able to find a suitably complete reference for this (see [L], [M], the appendix in [B], and, most recently, [CT1, p. 117, and the references therein]). The point with the sign lies in the map (7.4). For example, the candidate for this map which sends $[P] \in F^{1} \operatorname{Br}(X)$ to the extension class of the sequence $0 \rightarrow \operatorname{Pic}\left(X_{s}\right) \rightarrow \operatorname{Pic}\left(P_{S}\right) \rightarrow \mathbb{Z} \rightarrow 0$ requires, indeed, no sign in the formula.

Now let $i=2$ in (7.5). We show that a similar description holds, with $\Delta_{n}^{1}$ replaced by $\Delta_{n}^{2}$ and bearing in mind that we are committed here to work with rational coefficients. The pairing (7.5) for $i=2$ leads, by adjunction, to a morphism

$$
T(X)=F^{2} \mathrm{CH}_{0}(X) \longrightarrow \operatorname{Hom}_{\mathbb{Z}}(\operatorname{Br}(X), \operatorname{Br}(k))_{\mathbb{Q}} .
$$

On the other hand, the edge homomorphism $\operatorname{Br}(X) \rightarrow H^{0}\left(G_{k}, \operatorname{Br}\left(X_{S}\right)\right)$ and the cup-product map yield a morphism $\operatorname{Ext}_{G_{k}}^{2}\left(\operatorname{Br}\left(X_{s}\right), k_{s}^{*}\right)_{\mathbb{Q}} \rightarrow \operatorname{Hom}_{\mathbb{Z}}(\operatorname{Br}(X), \operatorname{Br}(k))_{\mathbb{Q}}$, whence, by composing with the inclusion $\operatorname{Br}\left(X_{s}\right) \hookrightarrow H^{2}\left(X_{s}, \mathbb{G}_{m}\right)$, a morphism

$$
\operatorname{Ext}_{G_{k}}^{2}\left(H^{2}\left(X_{s}, \mathbb{G}_{m}\right), k_{s}^{*}\right)_{\mathbb{Q}} \longrightarrow \operatorname{Hom}_{\mathbb{Z}}(\operatorname{Br}(X), \operatorname{Br}(k))_{\mathbb{Q}} .
$$

We then have the following.

THEOREM 7.8

Let $X$ be a smooth, projective, geometrically irreducible variety of dimension $n$ over a field $k$. In the above notation, the map (7.6) is the composition of the map $\Delta_{n}^{2}$ from (5.6) with the map (7.7).

\section{Proof}

Taking advantage once more of the use of rational coefficients, we show first that we may replace $k$ by any finite extension $k \subset k_{1}$, without loss of generality. Indeed, in the notation of (7.2) one has, denoting by a subindex ( $)_{1}$ the effect of base change from $k$ to $k_{1}$,

$$
\left\langle\left[z_{1}\right],\left[P_{1}\right]\right\rangle=\langle[z],[P]\rangle_{1} .
$$


It follows that we a have a commutative diagram

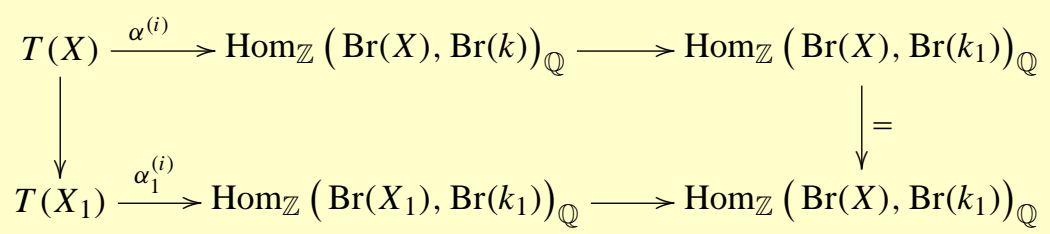

where $\alpha^{(i)}, i=1,2$, stand for the two maps to be compared and $\alpha_{1}^{(i)}, i=1,2$, are the corresponding maps over $k_{1}$. The remaining arrows bear the straightforward meaning. Then, writing $m=\left[k_{1}: k\right]$, we deduce by means of the norm map $N_{k_{1} / k}: \operatorname{Br}\left(k_{1}\right) \rightarrow$ $\operatorname{Br}(k)$ that if $[z] \in T(X)$ is such that $\alpha_{1}^{(1)}\left(\left[z_{1}\right]\right)=\alpha_{1}^{(2)}\left(\left[z_{1}\right]\right)$, then $m \alpha^{(1)}([z])=$ $m \alpha^{(2)}([z])$ and hence also $\alpha^{(1)}([z])=\alpha^{(2)}([z])$.

So, given $[z] \in T(X)$, we may assume, without loss of generality, that $z=$ $\sum_{i=1}^{r} n_{i} x_{i}$, with $k\left(x_{i}\right)=k$ for all $i$. Enlarging further the base field, we may assume also that the support $Z=|z|$ of $z$ is contained in a geometrically integral curve $C \subset X$ and that we have a finite set $S$ of geometrically integral curves on $X$ having $C$ as one of its members, such that the remaining curves do not meet $Z$ and such that the set $S_{s}$ of inverse images of these curves in $X_{s}$ is adequate for $Z$. As earlier in this paper, we write $Z_{s}$ for the 0 -dimensional closed subset of $X_{s}$, the inverse image of $Z$. Upon replacing $[z]$ by a positive integral multiple, we may assume that the morphism of $G_{k}$-modules

$$
H^{0}\left(Z_{s}, \mathbb{G}_{m}\right) / H^{0}\left(X_{s}, \mathbb{G}_{m}\right) \stackrel{z}{\longrightarrow} k_{s}^{*}
$$

(analogue of (2.3)) extends to a morphism of $G_{k}$-modules

$$
\operatorname{Pic}\left(X_{s}, Z_{s}\right) \stackrel{\tilde{z}}{\longrightarrow} k_{s}^{*} .
$$

Then $\Delta_{n}^{2}([z]) \in \operatorname{Ext}_{G_{k}}^{2}\left(\operatorname{Br}\left(X_{s}\right), k_{s}^{*}\right)$ is represented by the pushout along $\widetilde{z}$ of the exact sequence (see (3.14))

$$
\begin{gathered}
0 \longrightarrow \operatorname{Pic}\left(X_{s}, Z_{s}\right) \longrightarrow \prod_{C_{s}^{\prime} \in S_{s}} \operatorname{Pic}\left(C_{s}^{\prime}, Z_{s} \cap C_{s}^{\prime}\right) \\
\longrightarrow \widetilde{\operatorname{Br}}_{S_{s}}\left(X_{s}, Z_{s}\right) \longrightarrow \operatorname{Br}\left(X_{s}\right) \longrightarrow 0 .
\end{gathered}
$$

We note that, due to our particular choice of $S$, one actually has $\widetilde{\operatorname{Br}}_{S_{s}}\left(X_{s}, Z_{s}\right) \cong$ $\widetilde{\operatorname{Br}}_{S_{s}}\left(X_{s}\right)$ and $\prod_{C_{s}^{\prime} \in S_{s}} \operatorname{Pic}\left(C_{s}^{\prime}, Z_{s} \cap C_{s}^{\prime}\right)=\left(\prod_{C_{s}^{\prime} \in S_{s} \backslash\left\{C_{s}\right\}} \operatorname{Pic}\left(C_{s}^{\prime}\right)\right) \times \operatorname{Pic}\left(C_{s}, Z_{s}\right)$. Let $[P] \in \operatorname{Br}(X)$, and take its image $\left[P_{s}\right]$ in $H^{0}\left(G_{k}, \operatorname{Br}\left(X_{S}\right)\right)$. By means of explicit (Galois) cocycle manipulation, we compute the image of $\left[P_{S}\right]$ by the iterated connecting homomorphism for (7.12) and then the image of this by (7.11). (It should come as no surprise that only (7.10) is actually involved in this last stage of the computation.) We 
show that the so-obtained cocycle represents $\langle[z],[P]\rangle \in \operatorname{Br}(k)$, and this settles the proof of Theorem 7.8.

Fix, for all $C_{s}^{\prime} \in S_{s}$, an invertible sheaf $\xi_{s, C_{s}^{\prime}}$ on $P_{s, C_{s}^{\prime}}$ restricting to $\mathscr{O}(1)$ on the geometric fibres of $P_{s, C_{s}^{\prime}} \rightarrow C_{s}^{\prime}$. Then $\left(P_{s}, \xi_{s}\right)$ so constructed represents a lifting of $\left[P_{s}\right]$. The image of $\left[P_{s}\right]$ by the first connecting homomorphism is represented by the image of the 1-cochain which sends $\sigma \in G_{k}$ to the collection formed by the $\sigma_{\xi_{s, C_{s}^{\prime}}} \otimes \xi_{s, C_{s}^{\prime}}^{-1}$ for $C_{s}^{\prime} \neq C_{s}$, plus the couple formed by ${ }^{\sigma} \xi_{s, C_{s}} \otimes \xi_{s, C_{s}}^{-1}$ together with an (arbitrary) trivialization along $Z_{s} \subset C_{s}$. (Note that, for all $C_{s}^{\prime} \in S_{s}$, the sheaf $\sigma \xi_{s, C_{s}^{\prime}} \otimes \xi_{s, C_{s}^{\prime}}^{-1}$ is canonically isomorphic to the inverse image of its direct image in $C_{s}^{\prime}$, and therefore it can unambiguously be thought of as an invertible sheaf on $C_{s}^{\prime}$.) Now, choosing a trivialization of ${ }^{\sigma} \xi_{s, C_{s}} \otimes \xi_{s, C_{s}}^{-1}$ along $Z_{s}$ is tantamount to choosing a relative isomorphism

$$
\xi_{s, C_{s}} \otimes \mathscr{O}_{P_{s, Z_{s}}} \stackrel{\widetilde{\sigma}}{\longrightarrow} \xi_{s, C_{s}} \otimes \mathscr{O}_{P_{s, Z_{s}}}
$$

over the isomorphism $\sigma: P_{s, Z_{s}} \stackrel{\sim}{\longrightarrow} P_{s, Z_{s}}$. In this notation, the image of $\left[P_{s}\right]$ by the iterated connecting homomorphism for (7.12) is represented by the image in $\operatorname{Pic}\left(X_{s}, Z_{s}\right)$ of the 2-cocycle $\rho$ with values in $H^{0}\left(Z_{s}, \mathbb{G}_{m}\right)$ given by the formula $\rho(\sigma, \tau)=(\widetilde{\sigma \tau})^{-1} \widetilde{\tau} \widetilde{\sigma}$ for all $\sigma, \tau \in G_{k}$.

We show that the image of $\rho$ by the map $z$ in (7.10) represents $\langle[z],[P]\rangle \in$ $\operatorname{Br}(k)$. For all $i=1, \ldots, r$, choose a closed point $\tilde{x}_{i} \in P$ above $x_{i}$. Call $T_{s, i} \subset P_{s}$ the 0 -dimensional closed subset, inverse image of $\left\{\tilde{x}_{i}\right\}$. For simplicity, we write $P_{s, i}$ for $P_{s, x_{i, s}}=P_{x_{i}, s}$. We also write $\xi_{s, i}$ for the invertible sheaf $\xi_{s, C_{s}} \otimes \mathscr{O}_{P_{s, i}}$ on $P_{s, i}$. By Remark 4.11, $\left\langle\left[x_{i}\right],[P]\right\rangle \in \operatorname{Br}(k)$ is minus the image of $\left[\xi_{s, i}\right]$ by the iterated connecting homomorphism of the exact sequence

$$
0 \longrightarrow k_{s}^{*} \longrightarrow H^{0}\left(T_{s, i}, \mathbb{G}_{m}\right) \longrightarrow \operatorname{Pic}\left(P_{s, i}, T_{s, i}\right) \longrightarrow \operatorname{Pic}\left(P_{s, i}\right) \longrightarrow 0 .
$$

Fix, for all $i=1, \ldots, r$, a trivialization $\theta_{s, i}: \mathscr{O}_{T_{s, i}} \stackrel{\sim}{\longrightarrow} \xi_{s, i} \otimes \mathscr{O}_{T_{s, i}}$. Then $\left(\xi_{s, i}, \theta_{s, i}\right)$ so constructed represents a lifting of $\left[\xi_{s, i}\right]$. Borrowing from our previous notation, we now write, for all $\sigma \in G_{k}$,

$$
\xi_{s, i} \otimes \mathscr{O}_{T_{s, i}} \stackrel{\widetilde{\sigma}}{\longrightarrow} \xi_{s, i} \otimes \mathscr{O}_{T_{s, i}}
$$

for the restriction of (7.13) above $T_{s, i}$. This is a relative isomorphism over $\sigma: T_{s, i} \stackrel{\sim}{\longrightarrow}$ $T_{s, i}$. Let us denote also by

$$
\mathscr{O}_{T_{s, i}} \stackrel{\widetilde{\sigma}_{0}}{\longrightarrow} \mathscr{O}_{T_{s, i}}
$$

the canonical relative isomorphism over $\sigma: T_{s, i} \stackrel{\sim}{\longrightarrow} T_{s, i}$. In this notation, the image of $\left[\xi_{s, i}\right]$ by the first connecting homomorphism for (7.14) is represented by the image of the 1-cochain $\varpi_{i}$ with values in $H^{0}\left(T_{s, i}, \mathbb{G}_{m}\right)$ given by the formula $\varpi_{i}(\sigma)=$ 
$\theta_{s, i}^{-1} \widetilde{\sigma}^{-1} \theta_{s, i} \widetilde{\sigma}_{0}$ for all $\sigma \in G_{k}$. Thus the image of $\left[\xi_{s, i}\right]$ by the iterated connecting homomorphism for (7.14) is represented by the 2-cocycle $d \varpi_{i}$. Writing this out, we find that $\rho^{-1}=\left(d \varpi_{1}, \ldots, d \varpi_{r}\right)$, and the result follows.

To end, we include here a remark by J.-L. Colliot-Thélène on the fact that the use of rational coefficients in the statement of Theorem (7.8) is unavoidable in general. This partially answers the question mentioned in Remark 1.8 .

\section{Remark 7.17 (see [CT2])}

In general, there exists no map $T(X) \rightarrow \operatorname{Ext}_{G_{k}}^{2}\left(\operatorname{Br}\left(X_{s}\right), k_{s}^{*}\right)$ such that, when composed in the above sense with the natural map $\operatorname{Br}(X) \rightarrow \operatorname{Br}\left(X_{S}\right)^{G_{k}}$, it yields the composition $T(X) \subset A_{0}(X) \rightarrow \operatorname{Hom}(\operatorname{Br}(X), \operatorname{Br}(k))$.

One has, in fact, numerous examples of geometrically rational surfaces $X / k$ with $k$ a $p$-adic field, or even the field of real numbers, such that the map $A_{0}(X) \rightarrow$ $\operatorname{Hom}(\operatorname{Br}(X), \operatorname{Br}(k))$ is nonzero. (The simplest example is a cubic surface $X / \mathbb{R}$ such that $X(\mathbb{R})$ has two connected components.) For such a surface, the Albanese variety is trivial, whence $A_{0}(X)=T(X)$, and the geometrical Brauer group $\operatorname{Br}(\bar{X})$ is zero.

One can give similar examples with nonrational surfaces, in this case surfaces fibered in conics over a curve of genus at least 1 . One has then $\mathrm{Alb}_{X} \neq 0$, but always $\operatorname{Br}\left(X_{S}\right)=0$.

Acknowledgments. We are indebted to the referee for pointing out that our results, stated originally for surfaces, apply without change to varieties of arbitrary dimension and to the editor for requiring us to take this up in a revised version. We are grateful to J.-L. Colliot-Thélène for his permission to include here his Remark 7.17.

\section{References}

[B] S. BLOCH, On the Chow groups of certain rational surfaces, Ann. Sci. École Norm. Sup. (4) 14 (1981), 41 -59. MR 83e:14006 482

[CT1] J.-L. COLLIOT-THÉLÈNE, Principe local-global pour les zéro-cycles sur les surfaces réglées, J. Amer. Math. Soc. 13 (2000), 101 - 124. MR 2000d:14010 482

[CT2] , personal communication, 2001. 485

[CTS] J.-L. COLLIOT-THÉLÈNE, and J.-J. SANSUC, On the Chow groups of certain rational surfaces: A sequel to a paper of S. Bloch, Duke Math. J. 48 (1981), 421 - 447. MR 83e:14007 449, 462, 463

[GT] H. A. GILLET and R. W. THOMASON, "The $K$-theory of strict Hensel local rings and a theorem of Suslin" in Proceedings of the Luminy Conference on Algebraic K-Theory (Luminy, France, 1983), J. Pure Appl. Algebra 34, no. 2-3, North-Holland, Amsterdam, 1984, 241 -254. MR 86e:18014 449 
[G] M. L. GREEN, "Higher Abel-Jacobi maps" in Proceedings of the International Congress of Mathematicians (Berlin, 1998), Vol. II, Doc. Math. 1998, Extra Vol. II, Doc Math., Bielefeld, Germany, 1998, 267 -276. MR 99k:14012 447, 449, 476

[SGA2] A. GROTHENDIECK, Cohomologie locale des faisceaux cohérents et Théorèmes de Lefschetz locaux et globaux, Séminaire de Géométrie Algébrique du Bois-Marie 1962 (SGA 2), Adv. Stud. Pure Math. 2, North-Holland, Amsterdam, 1968. MR 57:16294 456

[Gr1] — - "Le groupe de Brauer, I: Algèbres d'Azumaya et interprétations diverses" in Dix exposés sur la cohomologie des schémas, North-Holland, Amsterdam, 1968, 46-66. MR 39:5586a 454, 455

[Gr2] _ - "Le groupe de Brauer, II: Théorie cohomologique" in Dix exposés sur la cohomologie des schémas, North-Holland, Amsterdam, 1968, 67-87.

MR 39:5586b 454

[Gr3] - "Le groupe de Brauer, III: Exemples et compléments" in Dix exposés sur la cohomologie des schémas, North-Holland, Amsterdam, 1968, 88-188.

MR 39:5586c 454, 455, 470

[J1] U. JANNSEN, Continuous étale cohomology, Math. Ann. 280 (1988), 207 - 245. MR 89a:14022 450, 451, 452

[J2] Mixed motives and algebraic K-theory, Lecture Notes in Math. 1400, Springer, Berlin, 1990. MR 91g:14008 451, 452

[J3] - "Motivic sheaves and filtrations on Chow groups" in Motives (Seattle, 1991), Proc. Sympos. Pure Math. 55, Part I, Amer. Math. Soc., Providence, 1994, 245-302. MR 95c:14006 447, 448

[K] S. L. KLEIMAN, "Algebraic cycles and the Weil conjectures" in Dix exposés sur la cohomologie des schémas, North-Holland, Amsterdam, 1968, 359-386.

MR 45:1920 449, 472

[L] S. LICHTENBAUM, Duality theorems for curves over p-adic fields, Invent. Math. 7 (1969), 120 - 136. MR 39:4158 447, 481, 482

[M] YU. I. MANIN, "Le groupe de Brauer-Grothendieck en géométrie diophantienne" in Actes du Congrès International des Mathématiciens (Nice, 1970), I, Gauthier-Villars, Paris, 1971, 401 -411. MR 55:356 447, 449, 481, 482

[Mi1] J. S. MILNE, Étale cohomology, Princeton Math. Ser. 33, Princeton Univ. Press, Princeton, 1980. MR 81j:14002 450, 454, 465, 473

[Mi2] - Arithmetic Duality Theorems, Perspect. Math. 1, Academic Press, Boston, 1986. MR 88e:14028 453, 473, 475

[Mit] B. MITCHELL, Theory of Categories, Pure Appl. Math. 17, Academic Press, New York, 1965. MR 34:2647

[R] W. RASKIND, Higher l-adic Abel-Jacobi mappings and filtrations on Chow groups, Duke Math. J. 78 (1995), 33 - 57. MR 96d:14009 448 
[S] J.-P. SERRE, Cohomologie galoisienne, 3d ed., Lecture Notes in Math. 5, Springer, Berlin, 1965. MR 34:1328 453, 462, 473

Facultad de Matemáticas, Universidad de Barcelona, Gran Via 585, 08007 Barcelona, Spain 